\title{
Article \\ The Altes Family of Log-Periodic Chirplets and the Hyperbolic Chirplet Transform
}

\author{
Donnacha Daly ${ }^{1, *(\mathbb{D})}$ and Didier Sornette ${ }^{2, *, t, \ddagger(\mathbb{D})}$ \\ 1 School of Computer Science and Information Technology, Lucerne University of Applied Sciences and Arts, \\ Suurstoffi 1, 6343 Rotkreuz, Switzerland \\ 2 Department of Management, Technology and Economics, ETH Zurich, Scheuchzerstrasse 7, \\ 8092 Zürich, Switzerland \\ * Correspondence: donnacha.daly@hslu.ch (D.D.); dsornette@ethz.ch (D.S.); Tel.: +41-41-228-2478 (D.S.) \\ + Current address: Institute of Risk Analysis, Prediction \& Management, Academy for Advanced \\ Interdisciplinary Studies, Southern University of Science and Technology (SUSTech), Shenzhen 518055, China. \\ $\ddagger$ Current address: Tokyo Tech World Research Hub Initiative (WRHI), Institute of Innovative Research, Tokyo \\ Institute of Technology, Tokyo 152-8550, Japan.
}

check for

updates

Citation: Daly, D.; Sornette, D. The Altes Family of Log-Periodic

Chirplets and the Hyperbolic Chirplet

Transform. Symmetry 2021, 13, 1922.

https://doi.org/10.3390/sym13101922

Academic Editor:Abraham A. Ungar

Received: 6 September 2021

Accepted: 1 October 2021

Published: 13 October 2021

Publisher's Note: MDPI stays neutral with regard to jurisdictional claims in published maps and institutional affiliations.

Copyright: (c) 2021 by the authors. Licensee MDPI, Basel, Switzerland. This article is an open access article distributed under the terms and conditions of the Creative Commons Attribution (CC BY) license (https:/ / creativecommons.org/licenses/by/ $4.0 /)$.
Abstract: This work revisits a class of biomimetically inspired waveforms introduced by R.A. Altes in the 1970s for use in sonar detection. Similar to the chirps used for echolocation by bats and dolphins, these waveforms are log-periodic oscillations, windowed by a smooth decaying envelope. Log-periodicity is associated with the deep symmetry of discrete scale invariance in physical systems. Furthermore, there is a close connection between such chirping techniques, and other useful applications such as wavelet decomposition for multi-resolution analysis. Motivated to uncover additional properties, we propose an alternative, simpler parameterisation of the original Altes waveforms. From this, it becomes apparent that we have a flexible family of hyperbolic chirps suitable for the detection of accelerating time-series oscillations. The proposed formalism reveals the original chirps to be a set of admissible wavelets with desirable properties of regularity, infinite vanishing moments and time-frequency localisation. As they are self-similar, these "Altes chirplets" allow efficient implementation of the scale-invariant hyperbolic chirplet transform (HCT), whose basis functions form hyperbolic curves in the time-frequency plane. Compared with the rectangular time-frequency tilings of both the conventional wavelet transform and the short-time Fourier transform, the HCT can better facilitate the detection of chirping signals, which are often the signature of critical failure in complex systems. A synthetic example is presented to illustrate this useful application of the HCT.

Keywords: wavelet transform; complex systems; discrete scale invariance; chirping; log-periodicity

\section{Introduction to Altes Chirps and the Hyperbolic Chirplet Transform}

Over the course of the 1970s, Richard A. Altes developed the theory behind a new family of waveforms with optimal Doppler tolerance for sonar applications. Inspired by mammalian acoustic echo-location calls such as those of bats and dolphins, their design evolved to a set of carefully parameterised hyperbolic chirps with useful time-frequency (TF) localisation properties [1-5]. Some time later, Patrick Flandrin and their colleagues at the French national center for scientific research (CNRS) exposed the close mathematical parallels between sonar-based target description using these chirps, and multi-resolution wavelet analysis [6]. The current work extends these previous, and somewhat neglected ideas, with three key contributions.

First, with a view to promoting the general flexibility and usefulness of the Altes chirps, we propose a re-parameterisation which is more intuitive for application purposes: The waveforms are specified in terms of centre-frequency, bandwidth and chirp-rate. In addition to familiarity, these parameters have the advantage of simplifying TF localisation requirements and the study of other signal properties, including symmetries within the 
waveform family. These parameters are more transparent than the original parameter-set proposed by Altes.

Second, building on [6] which considered admissibility conditions, the Altes waveforms are formalised as wavelets with infinite regularity and an infinite number of vanishing moments. Furthermore, they are self-similar over certain discrete scalings, dilations and shifts, enabling highly efficient implementation of a wavelet transform. We are led to coin this the Hyperbolic Chirplet Transform (HCT), whose mother wavelet is an Altes chirp. A low-complexity, discrete-time HCT is developed which offers arbitrarily close approximation to the continuous HCT. The result is a powerful and practical extension to the existing body of wavelet tools for signal analysis.

Our third contribution is the observation that hyperbolic chirps are also Log-Periodic (LP) in the time-domain. Log-periodicity is associated with the deep symmetry of discrete scale invariance in physical systems. Such signals abound in nature, often as precursors to phase transitions in the non-linear dynamics of complex systems [7]. Accordingly, from a practical perspective, log-periodic oscillations with an acceleration towards criticality can serve as indicators of an incipient bifurcation. The ability to detect LP-signatures has diverse applications, such as prediction of critical failures in mechanical systems [8], or detection of crowding behaviour in price dynamics during financial bubbles and preceding market crashes [9]. Examples beyond failure diagnostics include animal species identification via call recordings, commercial \& military radar, and there are many more. By making the bridge between the chirplet approach and the LP property, we extend the toolset for detecting criticality in complex systems, with the mature body of knowledge from wavelet theory.

The next section presents a review of background material relevant to the current work, including a deeper look at the motivations for and examples of transforms for detecting log-periodicity. A recap of the Altes waveform together with our re-parameterisation is provided in Section 3. The conditions under which it can be used as a wavelet in a HCT are presented in Section 4, along with a discussion of its wavelet properties, and how to perform parameter selection for various design criteria. Section 5 is an empirical look at the performance of the HCT in detecting LP-oscillations in noise. Derivations of the main results can be found in the appendices.

\section{Related Work and State-of-the-Art}

This section exposes in detail the reasons why log-periodicity is a fundamentally interesting signal characteristic in many different and seemingly unconnected processes, as well as some of the existing methods which have been proposed for its detection. Section 2.1 starts with the motivation for our work, which is primarily that log-periodic signals can be an indicator of inherent instability in complex systems. Sections 2.2 and 2.3, respectively, look at classical approaches to detection of chirping signals, such as the short-time Fourier transform and Constant-Q transforms. Since these have not specifically been designed for chirp detection, we look at transforms which have, such as the chirplet transform in Section 2.4 and the Mellin transform in Section 2.5. While the former are not tuned for hyperbolic chirping, the latter are. Unfortunately, this approach can be computationally prohibitive, which brings us to our proposal, the Hyperbolic Chirplet Transform. This is introduced briefly in Section 2.6, to be examined in detail in the remainder of the work.

\subsection{Motivation: Log Periodicity \& Discrete Scale Invariance}

There are many compelling reasons to seek out log-periodic oscillations in noisy timeseries, given their relative abundance in nature and in engineering systems [7]. To the best of our knowledge, LP-constructs first appeared in the 1960s for modelling shocks to layered systems [10] and the discrete hierarchy of vortices in hydrodynamic turbulence [11]. Soon after, log-periodicity was recognised in the self-similarity of propagating waves [12]. Around the same time, the renormalisation group theory of critical phenomena introduced solutions for the statistical mechanics of critical phase with complex critical exponents, 
characterised by log-periodicity [13-15]. Phase transitions occurring on hierarchical lattices have been shown to exhibit discrete scale invariance, with its signature of complex exponents and LP-oscillation [16-18].

A central result is that LP-signatures indicate that a system and/or its underlying physical mechanisms have a hierarchy of characteristic scales corresponding to discrete scale invariance. This provides important insights into the underlying physics, which may allow us to make forecasts of rupture such as earthquakes [19,20], mechanical failure [21] or the bursting of bubbles in financial markets $[9,22,23]$. In fact, any system with built in geometrical hierarchy will lead to log-periodicity, such as wave propagation in fractal systems [24], Ising and Potts models on hierarchical structures [25,26] and sandpile models on discrete fractal lattices [27].

More recently it has been found that chirped fibre Bragg gratings can provide useful temperature sensing for medical applications when the chirping is hyperbolic [28]. It has also been shown that Altes original application of hyperbolic chirping for target localisation remains a bountiful research area, as recently seen in [29], which demonstrates that forwards-backwards chirping exploiting bidirectional log-periodicity improves target velocity estimation. The approach is also practical-it has been shown experimentally that even a very low-complexity 1-bit implementation of hyperbolic chirping can provide good localisation results [30]. Given our growing understanding of the ubiquity of LP-signatures in complex systems, it is useful to equip ourselves with reliable tools for their extraction and diagnosis.

\subsection{Chirp Detection Using Adaptive Transforms}

Numerous studies have called out the need to use and/or identify these types of chirping signals, with mixed success in actually doing so. The authors of [31] suggest that feature extraction from non-trivial signals requires adaptive TF-distributions (TFDs) tailored to the signal, which they demonstrate for the medical analysis of knee-joint vibrations. Their recommendation is that such adaptive-TFDs should have the property of scale-invariance, but they implement matching pursuit using a Gaussian windowed Gabor tiling which does not. The work of [32] proposes an adaptive Meyer wavelet as an improvement on the short-time Fourier transform (STFT) for processing medical signals containing chirps. Similarly, in [33], the authors suggest that frequency-swept ultrasonic sonar chirping could be a useful method for non-contact bio-mechanical measurement, in this case, of the chest movements associated with breathing. However, they choose to implement a simpler solution using pseudo-random sequences. Classification of blue whale calls was undertaken in [34] using both a STFT and a wavelet packet transform. Significant effort was given to optimise window-types and -lengths for feature extraction, which could potentially have been avoided using a chirp-like TFD. The work of [35] uses an adaptive STFT to continuously match to fast-varying AM-FM signals. Their example shows that adaptive STFT significantly outperforms fixed-length STFT for detection of hyperbolic chirps in a synthetic signal very similar to the one we propose here in Section 5. A thorough overview of the development of such TF-analysis techniques can be found in $[36,37]$.

\subsection{Constant-Q Transforms}

Constant- $Q$ transforms are defined as a bank of bandpass filters which each have the same ratio of center frequency to bandwidth-a ratio known as the Q-factor [38]. While not directly designed for the detection of time-domain log-periodicity, Constant- $Q$ transforms are worth considering for their geometrically spaced frequency resolution, which originally found use in audio applications, because this is how musical octaves are spaced [39]. In fact, Altes showed (see Appendix A) that audio signals designed to be Constant-Q under echoing, would have optimal Doppler tolerance which is fundamental to the design of the Altes chirplet. Others have shown that Constant-Q transforms can be efficiently implemented, in conjunction with the Fourier transform. For example, 
Ref. [40] introduces the Constant-Q, non-Stationary Gabor Transform (CQ-NSGT) which is an invertible Constant-Q transform implemented with a Fast Fourier Transform (FFT) on slices of the signal under analysis. While this transform is demonstrably superior to a conventional STFT in the analysis of musical tones, it does not have chirping basis functions, and will therefore be inferior in the detection of chirping to transforms which are specifically matched to the target signal.

\subsection{Chirplet Transforms}

The idea of using chirps as wavelets, i.e., chirplets, was first introduced in [41,42], leading to the Gaussian chirplet and warblet transforms. A hardware implementation for bat-call detection was provided in [43]. An adaptive version of the Gaussian chirplet was designed to linearise curves in the TF-plane in [44]. This has been used for detection of bat echo-location signals and compared with the Gaussian wavelet transform, the STFT and the Wigner Ville Distribution (WVD) [45], noting that "Hyperbolic chirps probably can better model signals such as the sound of bat." .

Wavelet-based LP-detection methods were also compared in [46]. The Cauchy wavelet performed worst, the Mexican Hat better and the Morlet best. More comprehensive attempts to capture general non-linear TF modulations using the Polynomial Chirplet Transform were developed in [47] and applied to bat-sonar detection in [48]. None of these used a hyperbolic chirp in a wavelet transform for LP-detection. "Hyperbolic wavelets" do appear elsewhere, e.g., $[49,50]$ but with a different meaning. To avoid confusion, we refer exclusively to hyperbolic chirplets in the current context.

\subsection{Power Law Chirps and the Mellin Transform}

The hyperbolic chirp was introduced as a special case of the power law chirp in [51]. Its use for detection of chirping gravitational waves was presented in [52]. It is seen that while the optimal detection of a linear chirp is via the WVD, for power-law chirps the optimal detector uses the Bertrand distribution, which can be computationally prohibitive. As an alternative, the Mellin transform represents a signal as a projection onto a family of hyperbolic chirps, analogous to the Fourier transform projection on a basis of complex exponentials. This approach was applied to LP-detection in [53]. It was shown that the Mellin transform, coupled with a Fourier transform can be used to replace a wavelet transform, and is suited to problems of scale invariance. This idea was extended in [54] with the Fourier modified Mellin transform exploiting scale-invariance for improved waveform detection, including chirps. Similarly, in [55] the Mellin Transform has been used to provide the LP decomposition of the most general solution of the renormalisation group equation. The Hyperbolic Chirplet Transform introduced here implements a similar decomposition, while offering more explicit control over computational complexity for different applications.

\subsection{The Hyperbolic Chirplet Transform}

In [56], a strong case, and a powerful methodology, were both presented for tailoring wavelets to the signals being detected. While the example of LP-detection was examined, the study did not extend to using a dedicated hyperbolic chirplet for the purpose. In [7], a direct link was made between the functional form of the Altes wavelet (our Equation (A6)) and the renormalisation group encountered in [55,57]. The solution to the homogeneous Altes wavelet equation was thus identified as self-similar with discrete scale invariance. Self similar wavelets were introduced in [58] for fractal modulation. However, these were neither identified as hyperbolic, nor used for LP-detection. The work of [59] demonstrated that a filter-bank of frequency-offset hyperbolic chirps could be used for resilient radar detection of accelerating targets, but did not propose to implement this filter-bank as a wavelet transform. Generalised target detection was shown in [6] to share important features with wavelet decomposition. This qualitative work proposed the autocorrelation function of the Altes chirp as the analysing wavelet but did not demonstrate the utility of 
these results. This latter work [6] is a useful launch-point for the theoretical elements of the current exposition, in which we choose to implement the wavelet transform using the Altes chirp as mother wavelet. We call this the Hyperbolic Chirplet Transform (HCT), and will label our new parameterisation of the Altes waveform as the Altes chirplet.

\section{Method for Re-Parameterising the Altes Waveform}

This section returns to the original Altes waveform and proposes a useful reparameterisation. Section 3.1 together with Appendix A provide a quick recap of its mathematical origins as a parameterised bandpass chirp. Sections 3.2-3.4 express these original parameters in terms of the waveform center frequency, bandwidth and chirp-rate, respectively. This allows re-parameterisation in Section 3.5 of the Altes waveform in terms of these more familiar signal characteristics, a considerably more accessible and useful formulation. Section 3.6 provides some examples of these waveforms under different parameterisations, to demonstrate the flexibility of the new approach.

\subsection{Derivation of the Altes Waveform}

Altes' pioneering work on chirps showed that a TF-localised pulse which is periodic in the logarithm of progressing time has optimal Doppler tolerance [2,3]. Appendix A revisits their derivation of the chirp $U(\omega)$ in the frequency domain as a function of angular frequency $\omega$

$$
U(\omega)=A \omega^{v} \exp \left(-\frac{1}{2} \frac{\log ^{2} \omega}{\log k}\right) \exp \left(j 2 \pi c \frac{\log \omega}{\log k}\right) .
$$

The set of real constants $\{A, v, k, c\}$ in Equation (1) is the parameterisation of a family of waveforms. However, this parameterisation is not straightforward to work with from a waveform design perspective, which is why in the following, a more familiar set of parameters is presented. We will find that Equation (1) can be alternatively expressed as

$$
U(\omega)=\exp \left(-\kappa_{\mathcal{c}} \log ^{2} \frac{\omega}{\omega_{0}}\right) \exp \left(j 2 \pi \frac{\log \omega}{\log \lambda}\right), \quad \forall \omega>0
$$

where $\omega_{0}$ is chirp centre-frequency, $\lambda$ is chirp-rate, and $\kappa_{c}$ is a bandwidth parameter.

\subsection{Magnitude-Response and Center Frequency $\omega_{0}$ of the Altes Waveform}

Equation (1) represents a bandpass waveform and should therefore be specific to a center frequency $\omega_{0}$ and a pair of upper and lower cutoff frequencies $\omega_{c}^{ \pm}$, equivalent to some bandwidth $B$. Let the log-magnitude response be defined as

$$
M(\omega) \triangleq \log |U(\omega)| \stackrel{(1)}{=} \log A+v \log \omega-\frac{1}{2} \frac{\log ^{2} \omega}{\log k}
$$

and, without loss of generality, be specified with a unit passband response ( $0 \mathrm{~dB}$ gain). This yields a maximum response $M\left(\omega_{0}\right)=0$ at $M^{\prime}\left(\omega_{0}\right)=0$ from which we obtain

$$
\omega_{0}=k^{v} \Rightarrow A=k^{-v^{2} / 2}
$$

as previously noted in [6]. As illustrated in Figure 1a, the magnitude response $|U(\omega)|$ drops away from unity at $\omega_{0}$ to some lower level $K_{c}>0$ at cutoff frequency $\omega_{c}$ giving

$$
\begin{aligned}
& M\left(\omega_{c}\right) \stackrel{(3)(4)}{=} \quad v \log \omega_{c}-\frac{v}{2} \log \omega_{0}-\frac{v}{2} \frac{\log ^{2} \omega_{c}}{\log \omega_{0}} \\
& \stackrel{!}{=} \log \left(K_{c}\right) \quad, \quad 0<K_{c}<1 \text {. }
\end{aligned}
$$



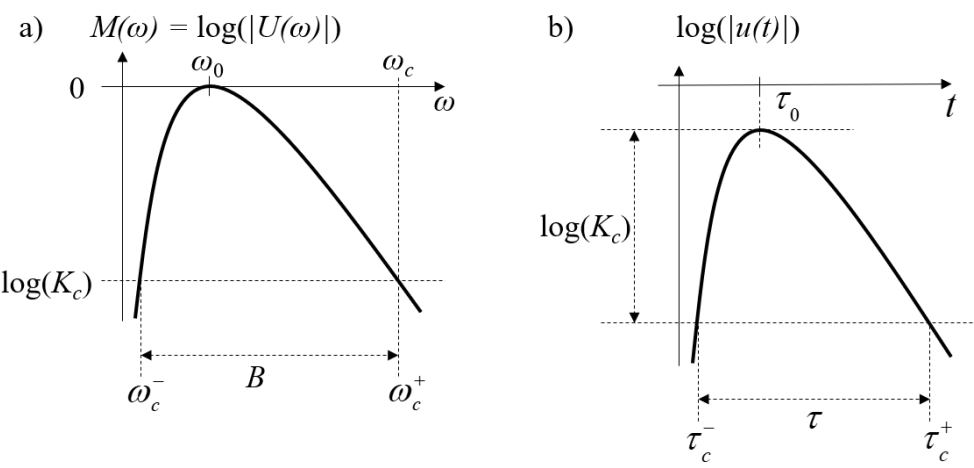

Figure 1. Re-parameterised Altes chirplet: (a) Log-magnitude frequency response defined in Sections 3.2 and 3.3 and (b) Log-magnitude time-domain envelope defined in Section 4.5.

By defining the positive constant

$$
\kappa_{c} \triangleq-\frac{\log K_{c}}{\log ^{2} \frac{\omega_{c}}{\omega_{0}}}>0
$$

we can easily re-parameterise the Altes constants $v$ and $k$ as

$$
v=2 \kappa_{c} \log \omega_{0} \text { and } k=\exp \left(\frac{1}{2 \kappa_{c}}\right)
$$

respectively. Substituting Equations (8) and (4) in Equation (3) gives the much simplified magnitude response centred around $\omega_{0}$

$$
M(\omega)=-\kappa_{c} \log ^{2} \frac{\omega}{\omega_{0}} .
$$

\subsection{Cutoff Frequency $\omega_{c}$ and Bandwidth B of the Altes Waveform}

The definition of $\kappa_{c}$ allows us to write

$$
\omega_{c}^{ \pm} \stackrel{(7)}{=} \omega_{0} \exp \left( \pm \sqrt{\frac{-\log K_{c}}{\kappa_{c}}}\right) \mathrm{rad} / \mathrm{s} .
$$

We can specify upper and lower cutoff frequencies by, respectively, choosing $\omega_{c}^{ \pm}$ greater or less than center frequency $\omega_{0}$. However, we recommend to speak only of the cutoff frequency $\omega_{c} \triangleq \omega_{c}^{+}>\omega_{0}$, defined as the upper one. This exerts control over decay of $U(\omega)$ at higher frequencies, and hence waveform smoothness (Section 4.2). There is less need to worry about the lower cutoff because of the infinite vanishing moments of $U(\omega)$ at $\omega=0$ causing rapid decay at low frequencies (Section 4.3). We obtain

$$
B \triangleq \omega_{c}^{+}-\omega_{c}^{-} \quad \stackrel{(10)}{=} \quad \omega_{0}\left(\frac{\omega_{c}}{\omega_{0}}-\frac{\omega_{0}}{\omega_{c}}\right) \mathrm{rad} / \mathrm{s}
$$

as the waveform bandwidth, with the lower cutoff frequency given by $\omega_{c}^{-}=\omega_{0}^{2} / \omega_{c}$.

For the presented formulation to be useful in practice, a value must be placed on $K_{c}=\left|U\left(\omega_{c}\right)\right|$ used in Equation (6). For the twin objectives of wavelet frequency localisation and avoidance of discrete-time aliasing elaborated in Section 4.8, it makes sense to place tight restrictions on $K_{c}$. We use a level corresponding to $-40 \mathrm{~dB}$ throughout, which of course can be varied depending on application requirements. This level implies

$$
20 \log _{10}\left|U\left(\omega_{c}\right)\right|=-40 \mathrm{~dB} \quad \Rightarrow \quad K_{c}=10^{-\frac{40}{20}}=0.01 .
$$




\subsection{Chirp Rate $\lambda$ of the Altes Waveform}

The Altes waveform can be found in the time-domain by inverse Fourier transform $u(t)=\mathcal{F}^{-1}\{U(\omega)\}$. This is shown in [60] to have phase response $\phi(t) \propto \log t$ which is a logarithmic function of time, i.e., a log-periodic chirp. Instantaneous frequency is

$$
\omega_{I}(t) \triangleq \frac{\mathrm{d} \phi(t)}{\mathrm{d} t} \propto \frac{1}{t}
$$

which describes a hyperbola in the time-frequency plane. Noting also that the instantaneous period $T_{I}(t)=2 \pi / \omega_{I}(t) \propto t$ is linear in time, chirping signals such as $u(t)$ are interchangeably referred to as having linear period modulation (LPM), hyperbolic frequency modulation (HFM) or logarithmic phase modulation [61]. They are also called log-periodic, which is preferred in the financial and physics literature [62].

As seen in Appendix A, the phase and magnitude response behaviours of the original Altes' waveform in Equation (1) are governed by single parameter $k$. In order to decouple them, and allow for simpler chirplet design, we introduce chirp rate $\lambda>0$ by

$$
\log \lambda \triangleq \frac{\log k}{c} .
$$

\subsection{Our Re-Parameterisation of the Altes Waveform}

The proposed re-parameterisation now emerges from Equations (1), (4), (6) and (14) as

$$
U(\omega)=\exp \left(\log K_{c} \frac{\log ^{2} \frac{\omega}{\omega_{0}}}{\log ^{2} \frac{\omega_{c}}{\omega_{0}}}\right) \exp \left(j 2 \pi \frac{\log \omega}{\log \lambda}\right), \quad \forall \omega>0
$$

or more concisely as in Equation (2). In contrast to the original Fourier domain specification of $U(\omega)$ [3], we propose to zero the non-positive frequencies and define

$$
U(\omega) \triangleq 0, \quad \forall \omega \leq 0 .
$$

The waveform $U(\omega)$ remains continuous at $\omega=0$ since

$$
\lim _{\omega \rightarrow 0^{+}} U(\omega)=\lim _{\omega \rightarrow 0^{-}} U(\omega)=U(0)=0 .
$$

While Altes preferred to impose Hermitian symmetry on $U(\omega)$ to ensure a real timedomain wavelet $u(t)$, preservation of the analytic form in Equations (15) and (16) retains useful phase and envelope properties. Our approach allows useful definitions for instantaneous amplitude and frequency when analysing arbitrary complex signals [51].

For a target threshold $K_{c}$, below which the frequency response is negligible, the Altes chirplet can now be parameterised from Equation (15) by the triplet $\left\{\omega_{0}, \omega_{\mathcal{c}}, \lambda\right\}$, i.e., center frequency, cutoff frequency and chirp-rate. This re-parameterisation exposes $\lambda$ as the scaling ratio of a discrete scale invariance symmetry, i.e., the ratio of the local periods of successive oscillations in the chirp. As the chirp rate parameter $\lambda \rightarrow 1$ in Equation (15), the oscillations become faster and faster until a singularity occurs at $\lambda=1$. To avoid it, we must consider $0<\lambda<1$ or $\lambda>1$. In fact, it is easy to show that we need only consider the parameter range $\lambda \in(0,1)$ and that the results for $\lambda \in(1, \infty)$ are identical, and found by reciprocation of $\lambda$. To see this, note that replacing $\lambda$ by $\frac{1}{\lambda}$ simply changes the sign of the complex exponential in Equation (15), equivalent to frequency-domain conjugation. This in turn represents time reversal and conjugation of the chirplet in the time-domain. Making $\lambda$-dependence explicit by subscript we obtain

$$
u_{\frac{1}{\lambda}}(t)=u_{\lambda}^{*}(-t) .
$$


An example of this effect is demonstrated below by comparing Figure 4 with Figure 5 . We benefit from this insight in Section 4.9 when choosing practical bounds for the chirp rate parameter $\lambda$.

\subsection{Selected Parameterisations of the Altes Waveform for Illustrative Purposes}

The goal of this section is to show how the center frequency, bandwidth and chirprate of the Altes waveform can be easily tuned under our new re-parameterisation. Four examples are presented. The first is an original parameterisation from Altes. The second is a tuning of the bandwidth and centre-frequency. The third is a tuning of the cutoff frequency. The fourth and final example illustrates the effect of an inverted chirp rate. In each case, we show the analytic time-domain waveform $u(t)$ as well as magnitude and phase responses of $U(\omega)$ from Equation (2).

Figure 2 recreates the original Altes waveform [3] with $\{v, k, c\}=\{-0.55,1.8,-0.35\}$. A primary difference is that our version of the chirp is complex, and both the imaginary component and complex envelope can be observed in addition to the real waveform. There is no frequency normalisation, and it is not obvious how the chosen parameters relate to the observed chirp. According to our new parameterisation, we can evaluate from Equation (4) that this bandpass waveform has center frequency $\omega_{0}=0.733 \mathrm{rad} / \mathrm{s}$, as well as providing from Equations (14), (10) and (11), the previously unavailable chirp rate $\lambda=0.187$, cutoff frequency $\omega_{c}=7.41 \mathrm{rad} / \mathrm{s}$, and bandwidth $B=7.34 \mathrm{rad} / \mathrm{s}$, assuming a $-40 \mathrm{~dB}$ cutoff, limit, i.e., $K_{c}=10^{-2}$.
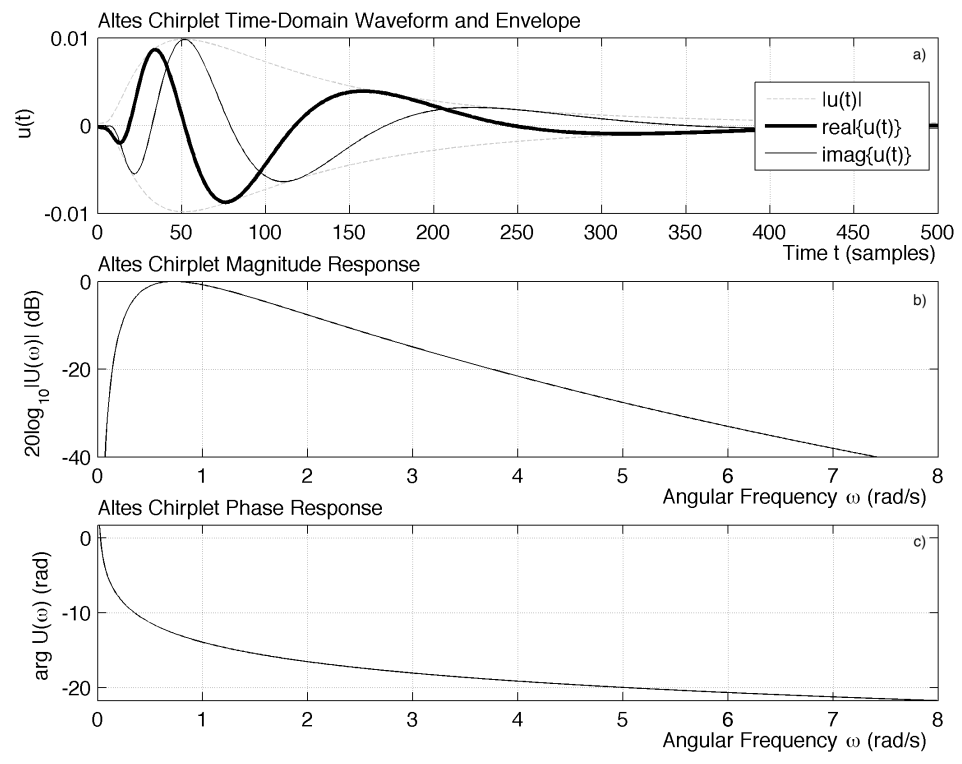

Figure 2. Waveform identical to that of the original Altes paper [3] using their parameter set $\{v, k, c\}=\{-0.55,1.8,-0.35\}$. (a) Time domain chirp (b) Frequency domain magnitude response and (c) Frequency domain phase response. See our first example in Section 3.6 for details.

In the second example, we choose to show how the new parameterisation of Equation (15) allows practical specification of centre-frequency, bandwidth (via Equation (11)) and chirp rate, in this case $\left\{\omega_{0}, B, \lambda\right\}=\left\{\frac{\pi}{6}, \frac{\pi}{5}, \frac{3}{4}\right\}$. In Figure 3, it can be seen that the $-40 \mathrm{~dB}$ bandwidth $B$ is $0.2 \pi$ as specified. When implementing in discrete time, it is useful to work in units of normalised frequency, such that sampling frequency $\omega_{s}=2 \pi$ corresponds to a unit sampling interval. This convention is adopted in the remainder, which fixes the Nyquist rate at $\omega_{\mathrm{Nyq}}=\pi$ for the coming discussion on discrete-time implementation.

Our third example illustrates the tuning of waveform cutoff frequency using parameters $\left\{\omega_{0}, \omega_{c}, \lambda\right\}=\left\{\frac{\pi}{10}, \frac{9 \pi}{10}, \frac{3}{4}\right\}$. In Figure 4 , it can be seen that the $-40 \mathrm{~dB}$ cutoff is tightly tuned to $\omega_{c}=\frac{9 \pi}{10}$ as specified. This tight control of magnitude response decay at high fre- 
quencies can be used to influence aliasing in a discrete time implementation. Furthermore, the wider bandwidth in this example opens a greater range of frequencies over which to chirp, as compared with Figure 3.

A final example is chosen to show the effect of reciprocation of chirp rate parameter $\lambda$. From Equation (2), it is clear that $0<\lambda<1$ results in negative phase response, while $\lambda>1$ yields positive phase response. We showed in Section 3.5 that replacing $\lambda$ by $\frac{1}{\lambda}$ amounts to conjugation and time reversal of the Altes chirp. The effect is clear to see in Figure 5, in which the Altes waveform is parameterised identically to the previous example, with the exception of the chirp rate, which is inverted: $\left\{\omega_{0}, \omega_{c}, \lambda\right\}=\left\{\frac{\pi}{10}, \frac{9 \pi}{10}, \frac{4}{3}\right\}$. The effects of chirp-rate on wavelet delay-spread will be examined further in Section 4.6 on time-frequency localisation.
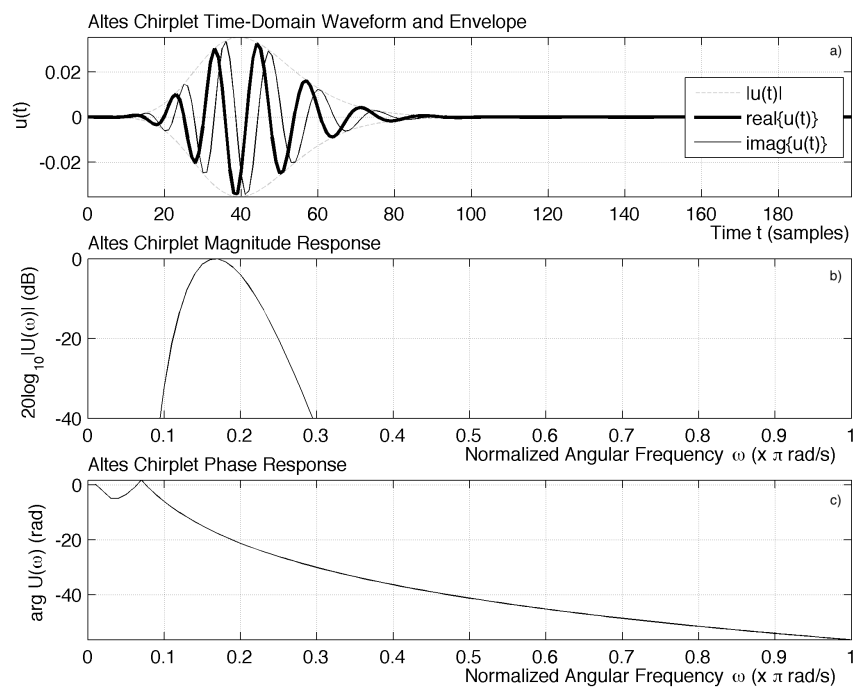

Figure 3. Re-parameterised Altes waveform with $\left\{\omega_{0}, B, \lambda\right\}=\left\{\frac{\pi}{6}, \frac{\pi}{5}, \frac{3}{4}\right\}$. (a) Time domain chirp (b) Frequency domain magnitude response and (c) Frequency domain phase response. See the second example in Section 3.6 for details.
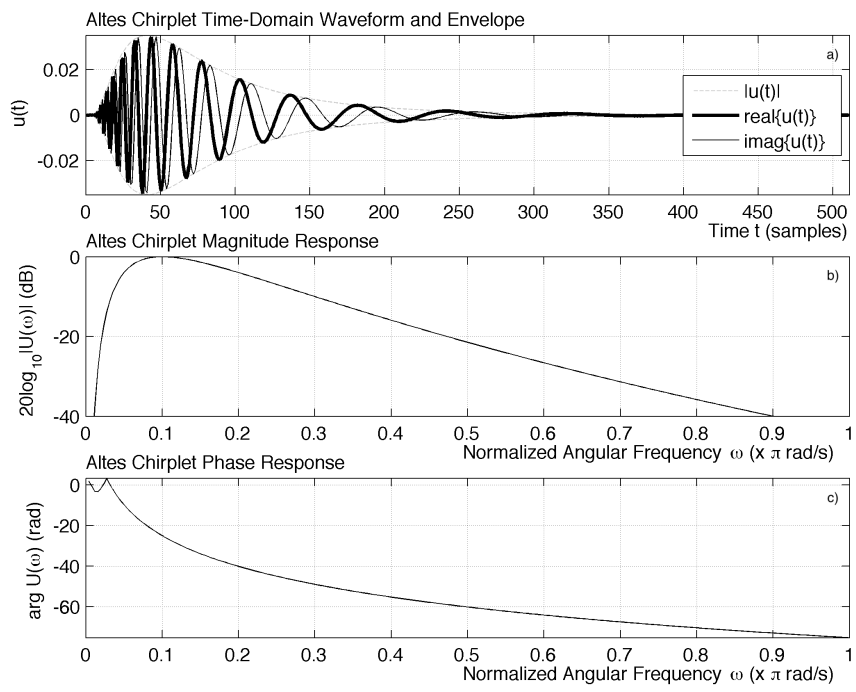

Figure 4. Re-parameterised Altes waveform with $\left\{\omega_{0}, \omega_{c}, \lambda\right\}=\left\{\frac{\pi}{10}, \frac{9 \pi}{10}, \frac{3}{4}\right\}$. (a) Time domain chirp (b) Frequency domain magnitude response and (c) Frequency domain phase response. See the third example in Section 3.6 for details. 

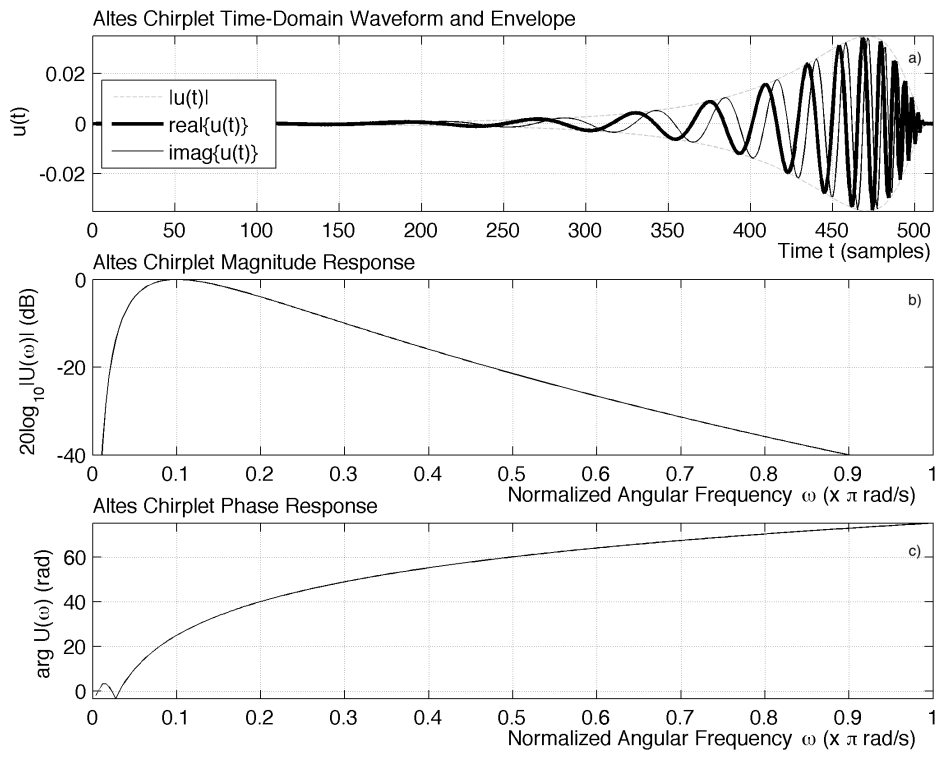

Figure 5. Re-parameterised Altes waveform with $\left\{\omega_{0}, \omega_{c}, \lambda\right\}=\left\{\frac{\pi}{10}, \frac{9 \pi}{10}, \frac{4}{3}\right\}$. (a) Time domain chirp (b) Frequency domain magnitude response and (c) Frequency domain phase response. See the fourth example in Section 3.6 for details.

\section{Method for Selecting the Parameters of the Altes Chirplet}

Up to this point, we have arrived at the description of a family of waveforms reparameterised in Equation (2) from Altes' original frequency-domain representation. In Section 4.1, it is shown that we are actually dealing with a family of wavelets in the formal (admissible) sense. We extend our understanding of these chirping wavelets, or chirplets, by showing that they also have additional desirable properties such as infinite regularity (Section 4.2) and an infinite number of vanishing moments (Section 4.3). Furthermore, and quite remarkably, Section 4.4 shows that the self-similar structure of the Altes wavelet can be exploited for efficient implementation within a continuous wavelet transform. which we label the Hyperbolic Chirplet Transform (HCT). Sections 4.5-4.11 deal with design criteria, and the selection of Altes wavelet parameters $\left\{\omega_{0}, \omega_{c}, \lambda\right\}$ for different applications and implementations of the HCT.

\subsection{Admissibility}

The continuous wavelet transform (CWT) of a real or complex, square integrable signal $s(t)$ at position $b$ and scale $a$ is

$$
C_{\psi}(a, b)=\int_{\mathbb{R}} s(t) \frac{1}{\sqrt{a}} \psi^{*}\left(\frac{t-b}{a}\right) \mathrm{d} t, \quad a \in \mathbb{R} \backslash\{0\}, b \in \mathbb{R}
$$

where $\psi(t)$ is said to be an admissible wavelet if it satisfies the fairly loose conditions that it is square integrable and sufficiently band-limited [63,64]. For real wavelets, it is sufficient for admissibility to define the CWT for only positive scalings $a \in \mathbb{R}^{+}$. However, in Equation (16) we design the Altes wavelet to be a complex analytic waveform. In this case, to ensure an injective transform in Equation (19), we should allow also for negative scalings $a \in \mathbb{R} \backslash\{0\}$, following the ideas in [63]. When $\psi(t)$ is an admissible wavelet, Calderón's reproducing identity tells us that, in this case, the original signal $s(t)$ can be recovered exactly from its wavelet coefficients $C_{\psi}(a, b)$ by the inverse transform

$$
s(t)=\frac{1}{\mathcal{C}_{\Psi}} \int_{\mathbb{R}} \int_{\mathbb{R} \backslash\{0\}} C_{\psi}(a, b) \frac{1}{\sqrt{a}} \psi\left(\frac{t-b}{a}\right) \mathrm{d} a \mathrm{~d} b,
$$


where $\mathcal{C}_{\Psi}$ is the admissibility constant. The formal admissibility conditions on $\psi(t)$ are

$$
\mathcal{E}_{\psi} \triangleq \int_{\mathbb{R}}|\psi(t)|^{2} \mathrm{~d} t<\infty \quad \text { and } \quad \mathcal{C}_{\Psi} \triangleq \int_{\mathbb{R}} \frac{|\Psi(\omega)|^{2}}{|\omega|} \mathrm{d} \omega<\infty
$$

corresponding to square-integrability and transform invertibility, respectively. $\mathcal{E}_{\psi}$ is the wavelet energy and we have used the Fourier transform $\mathcal{F}\{\psi(t)\}=\Psi(\omega)$.

Equation (19) represents correlation of $s(t)$ with scaled and shifted versions of the wavelet

$$
\psi_{a, b}(t)=\frac{1}{\sqrt{a}} \psi\left(\frac{t-b}{a}\right) .
$$

The factor $\frac{1}{\sqrt{a}}$ ensures that $\left\|\psi_{a, b}(t)\right\|$ is independent of $\{a, b\}$, and often the mother wavelet $\psi(t)$ is normalised so that

$$
\mathcal{E}_{\psi}=\|\psi(t)\|_{2}=\left\|\psi_{a, b}(t)\right\|_{2}=1 .
$$

The admissibility conditions tell us that $\psi(t)$ is a finite energy pulse with a frequency response that decays at high frequencies, and which must have no DC component. In simpler words, it is a frequency localised bandpass waveform.

Proposition 1. The Altes waveform $u(t)=\mathcal{F}^{-1}\{U(\omega)\}$ is an admissible wavelet.

A proof is given in Appendix B.1, allowing us to correctly characterise the Altes waveform as a wavelet. Furthermore, since it is a log-periodic chirp, we can refer to it as the Altes chirplet, and its application within a CWT as the Hyperbolic Chirplet Transform, consistent with prior taxonomy. Wavelets that only have the property of admissibility are known as crude wavelets [65], because admissibility is a weak condition and does not always result in usefulness for real applications. This leads us to examine other wavelet properties which can enhance their applicability.

\subsection{Regularity}

Regularity describes the smoothness of a wavelet $\psi(t)$ in the time-domain. The order of regularity corresponds to the number of times $\psi(t)$ is continuously differentiable. We say that $\psi(t)$ is bounded and has uniform Lipschitz regularity of order $\alpha>0$ over $\mathbb{R}$ if its frequency weighted magnitude response is Lebesgue integrable according to

$$
\int_{\mathbb{R}}|\Psi(\omega)|\left(1+|\omega|^{\alpha}\right) \mathrm{d} \omega<\infty .
$$

This captures the fact that smoothness in time is directly related to the rate of decay at high frequencies. Intuitively, signals with higher frequency content vary more rapidly, and are therefore less regular.

Proposition 2. The Altes chirplet $u(t)$ has infinite regularity.

A proof is given in Appendix B.2. Wavelets are often used for compression applications, in which signals are represented by a truncated set of CWT coefficients. Regular wavelets have the advantage that the undesirable artefacts arising from truncation (e.g., audio distortion) are less noticeable when compared to those produced using less smooth wavelets, even if the compression error magnitude is similar. Infinitely regular wavelets are thus suitable for use in information-coding. The Altes chirplet joins an august family including the Morlet, Mexican Hat, Meyer, Gauss and Shannon wavelets, all infinitelycontinuously differentiable in time. 


\subsection{Vanishing Moments}

If wavelet $\psi(t)$ has $M$ vanishing moments, then it is orthogonal to all polynomials of order $M-1$. A richer set of signals can be represented with a sparser set of coefficients when the mother wavelet of the analysing CWT has higher number of vanishing moments. This the highest integer $M$ such that in the frequency-domain

$$
\left.\frac{\mathrm{d}^{m} \Psi(\omega)}{\mathrm{d} \omega^{m}}\right|_{\omega=0}=0 \forall m \in\{0,1,2, \ldots, M\} .
$$

This makes it clear that vanishing moments flatten the wavelet response $\Psi(\omega)$ around the zero frequency. However, steeper response decay at low frequencies narrows the wavelet bandwidth from below, and there must be a corresponding time dilation. Thus, a higher number of vanishing moments comes at the cost of increasing support in the time-domain, i.e., longer wavelets. This will be addressed in Section 4.5.

Proposition 3. The Altes chirplet has an infinite number of vanishing moments.

A proof is provided in Appendix B.3.

\subsection{Scale Invariance}

When the Altes Chirplet is used as the mother wavelet in the CWT, we call this the Hyperbolic Chirplet Transform (HCT). The self-similarity of the Altes Chirplet, as it emerges from the homogeneity of Equation (A6), leads to the extraordinary property of transform scale invariance, i.e., the HCT can, in theory, be computed trivially at any scale, from knowledge of one scale only. In practise, finite precision and sampling effects limit the extent to which this can be exploited in real systems.

Proposition 4. The HCT is scale invariant.

Appendix B.4 provides a proof, with the implication that the HCT can be implemented as an extremely efficient transform as a result of the self-similarity of the Altes chirplet. In theory the Hyperbolic Chirplet Transform $C_{u}(a, b)$ can be computed once at scale $a$, and the coefficients $C_{u}(m a, b)$ can be found at all other wavelet scales $m a$ via Equation (A29). In practice, the HCT is implemented digitally, with the waveform being discretised in both time and amplitude. As such a full, infinite-support, infinite-scale, multi-resolution analysis will not be achievable, but will depend on the specific implementation.

\subsection{The Delay Spread of the Altes Chirplet}

Altes chirplets were shown in Section 4.3 to have an infinite number of vanishing moments. This requires infinite time-domain support, suggesting very long wavelets. We can ask, how does Altes chirplet delay-spread $\tau$ depend on the parameterisation $\left\{\omega_{0}, \omega_{c}, \lambda\right\}$ of Equation (15), and how does it trade off against chirplet bandwidth $B$ ? Indeed, what are useful values of, or limits on these parameters for signal processing applications? To answer this, we start by defining the delay-spread in a manner similar to how bandwidth is specified in the frequency-domain in Section 3.2. Time localisation implies that the time-domain envelope $|u(t)|$ rises gradually from zero to some peak at $t=\tau_{0}$ where the waveform energy is concentrated, before falling away again to zero, as conceptualised in Figure $1 b$, and exemplified in Figures $2-5$. We define delay-spread empirically as the time $\tau$ between the earliest appearance of significant energy at $t=\tau_{c}^{-}$, and the later time $t=\tau_{c}^{+}$ after which the energy appears to have vanished: $\tau=\tau_{c}^{+}-\tau_{c}^{-}$. For this to be precisely specified, some threshold amplitude is needed, which defines the presence or absence of wave energy at cutoff points $\tau_{c}^{ \pm}$. We use the same $-40 \mathrm{~dB}$ level of Equation (12) used in 
defining the waveform bandwidth in Section 3.2. Normalising to the peak magnitude of the time-domain waveform, we define this amplitude threshold as

$$
\frac{\left|u\left(\tau_{c}^{ \pm}\right)\right|}{\left|u\left(\tau_{0}\right)\right|} \stackrel{!}{=} K_{c}=0.01
$$

While this is not the most conventional definition of delay spread, it provides consistency with Equation (12) for time-frequency localisation analysis, and is certainly valid.

\subsection{Choosing Altes Parameters for Efficient Time-Frequency Localisation}

As there is no trivial time-domain representation of the Altes chirplet, this part of the study is numerical. We are going to do an empirical sweep over the parameters $\left\{\omega_{0}, \omega_{c}, \lambda\right\}$ to observe their influence on delay spread $\tau$ and bandwidth $B$. In Section 3.4, it was seen that investigation of $\lambda \in(0,1)$ is sufficient. All derived results then also apply for $1 / \lambda$, i.e., $\lambda \in(1, \infty)$. Furthermore, as outlined in Section 3.6, we consider unit sampling and need only consider $\omega_{0} \in(0, \pi)$ and $\omega_{c} \in\left(\omega_{0}, \pi\right]$. The results of a dense parameter sweep within these domains are presented in Figure 6, showing the measured delay spread and bandwidth for each grid point, i.e., each dot represents one instance of the Altes chirplet $u(t)$ at a given value of the parameter set $\left\{\omega_{0}, \omega_{c}, \lambda\right\}$ in Equation (15). It can be immediately seen that there is an efficient frontier shown by the thick line to the lower left, along which there is an optimal trade-off between time-localisation and frequency-localisation. Altes chirplets away from this Frontier are inefficient, as they could be tuned to have smaller bandwidth without increasing the delay-spread and vice versa.

Examining the Altes parameters along this curve, we find in all cases that $\lambda<1 / 2$, a loose parameter bound for efficiency. This is because the chirplet in Equation (15) is ill-behaved near $\lambda=1$ and is certainly not efficiently localised there. The effects of this ill-behaviour begin to disappear for $\lambda<1 / 2$. At the upper left of the frontier, $\omega_{0}>\pi / 2$ and $B<\pi / 4$, and the waveform becomes narrow-band. Chirping seems impossible, and we appear to be dealing with a conventional wavelet, rather than a chirplet.

Moving down the curve to the right, it is found that the cutoff frequency quickly converges to the Nyquist rate, $\omega_{c} \rightarrow \pi$, while the center frequency slides down to $\omega_{0} \rightarrow$ $\pi / 2$, as the Altes chirplet becomes less frequency localised. Finally, the efficient frontier flattens out at $B=3 \pi / 4$. This is the point of minimum delay spread, which occurs for chirplets with $\left\{\omega_{0}, \omega_{c}\right\}=\{\pi / 2, \pi\}$, consistent with Equation (11). Table 1 summarises the efficient parameterisations discussed in this section.

Table 1. Parameterisations of the Altes Chirplet for efficient time-frequency localisation. The parameter bounds are explained in detail Section 4.6.

\begin{tabular}{lccc}
\hline Localisation & $\omega_{0}$ & $\omega_{c}$ or $\boldsymbol{B}$ & $\lambda$ \\
\hline $\begin{array}{l}\text { Efficient frequency localisation } \\
\text { (minimal chirping) }\end{array}$ & $\omega_{0}>\frac{\pi}{2}$ & $\begin{array}{c}\omega_{c}<\pi \\
\left(B<\frac{\pi}{4}\right)\end{array}$ & $\begin{array}{c}\lambda<\frac{1}{2} \\
(\Leftrightarrow \lambda>2)\end{array}$ \\
\hline $\begin{array}{l}\text { Efficient time-freq. localisation } \\
\text { (the frontier) }\end{array}$ & $\omega_{0}>\frac{\pi}{2}$ & $\begin{array}{c}\omega_{c}=\pi \\
\left(\frac{\pi}{4}<B<\frac{3 \pi}{4}\right)\end{array}$ & $\begin{array}{c}\lambda<\frac{1}{2} \\
(\Leftrightarrow \lambda>2)\end{array}$ \\
\hline $\begin{array}{l}\text { Efficient time localisation } \\
\text { (min. delay-spread) }\end{array}$ & $\omega_{0}=\frac{\pi}{2}$ & $\begin{array}{c}\omega_{c}=\pi \\
\left(B=\frac{3 \pi}{4}\right)\end{array}$ & $\begin{array}{c}\lambda<\frac{1}{2} \\
(\Leftrightarrow \lambda>2)\end{array}$ \\
\hline
\end{tabular}




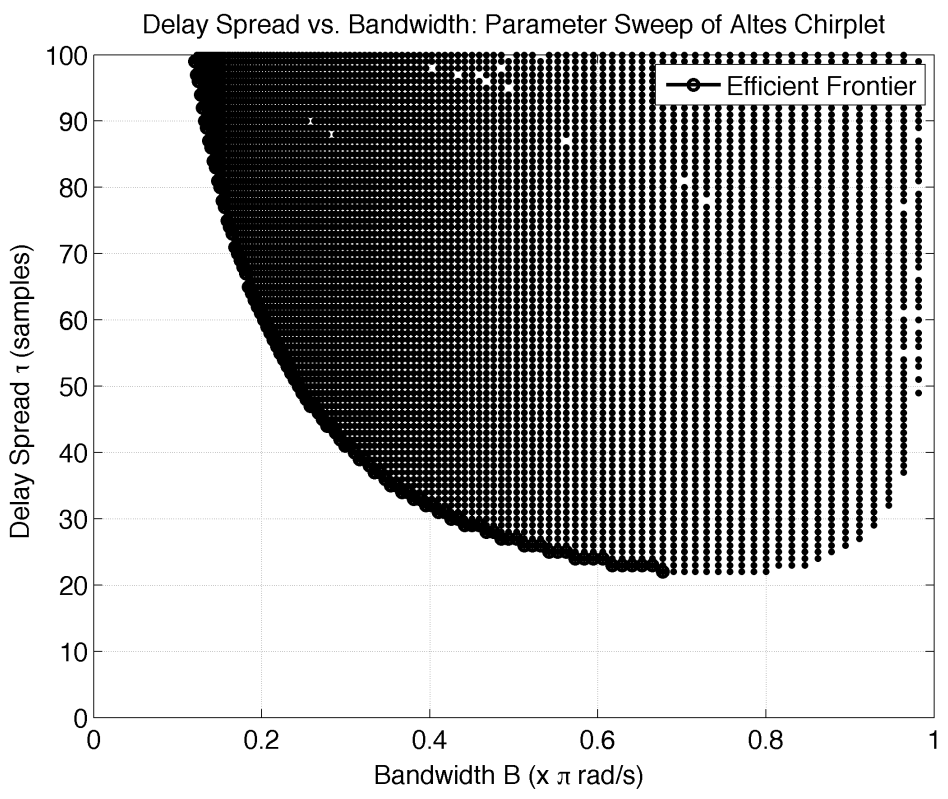

Figure 6. Visualisation of the measured bandwidth and delay spread of the Altes chirplet when sweeping over its parameter space $\left\{\omega_{0}, \omega_{c} \lambda\right\}$. See Section 4.6 for details.

\subsection{The Surprising Desirability of Inefficiently Localised Chirplets}

At this point, we could call it a day, having identified parameterisations of the Altes Chirplet for efficient TF-localisation. However, it appears that nature values chirping flexibility above localisation-efficiency when it comes to ubiquitous log-periodicities. For example, the parameterisation in Figure 2, while found to be a good model for bat chirps, is not sufficiently TF-localised to lie on the efficient frontier of Figure 6.

More generally, we have found it desirable (a) to select chirps away from the frequency localised regime, such that there is a sufficient range of frequencies over which to sweep; and similarly, (b) that the chirplet must be long enough lived in time to afford numerous oscillations of the log periodicity. Excessive time localisation impedes desirable chirping. Experience and research have taught us that the chirp waveforms encountered in practice, such as those used by animals for echolocation, as well as those encountered in the signatures of discrete-scale invariance such as fracture dynamics, exhibit inefficient time-frequency localisation. In simpler words, we seek chirplets with relatively wide bandwidths and long delay spreads, whose parameterisations are selected away from the efficient frontier of Figure 6. Supporting this idea, it has recently been shown that wide-band hyperbolic chirping significantly improves Doppler tolerance over narrowband chirping [66]. In fact, this work shows that even a linear chirp is seen to perform better than a hyperbolic narrowband chirp. The next sections examine how we might select parameters for such inefficiently TF-localised Altes chirplets.

\subsection{Choosing Center- and Cutoff-Frequency of Inefficiently Localised Altes Chirplets}

In Section 4.6, it was found that fixing cutoff frequency $\omega_{c}=\pi$ is required for efficient localisation. Now that inefficiently localised chirps are also in consideration, there remains a good reason to keep $\omega_{c}=\pi$. In order to minimise implementation complexity the chirplet should be represented by a sampling that is sparse, while ensuring that chirplet dynamics are fully captured without aliasing. Assuming a unit sampling interval (a sampling frequency of $\omega_{s}=2 \pi$ ), this translates directly to the requirement that the chirplet cutoff equals the Nyquist sampling rate $\omega_{c}=\omega_{\mathrm{Nyq}}=\pi$. This, together with Section 4.7 suggests to choose $\omega_{0}<\frac{\pi}{4}$ (i.e., $B>\frac{3 \pi}{4}$ ) for frequency delocalisation, i.e., such that wide-band chirping can occur. This constraint is captured in Table 2. 
Table 2. Parameter Bounds on the Altes Chirplet for Discrete-Time Implementation and use in the HCT.

\begin{tabular}{lcl}
\hline Parameter & Value(s) & Comment \\
\hline Center Frequency $\omega_{0}$ & $\omega_{0}<\frac{\pi}{4}$ & Wideband chirping \\
\hline Cutoff Frequency $\omega_{c}$ & $\omega_{c}=\omega_{\mathrm{Nyq}}=\pi$ & Critical-rate unit-sampling \\
\hline Chirp-Rate $\lambda$ & $1 / 4<\lambda<3 / 4$ & Bounded no. of oscillations \\
& $(\Leftrightarrow 4 / 3<\lambda<4)$ & \\
\hline Fourier Transform Size $N_{\mathrm{fft}}$ & Use Figure 7 to select & $N_{\mathrm{fft}}=1024$ works in most cases \\
\hline
\end{tabular}

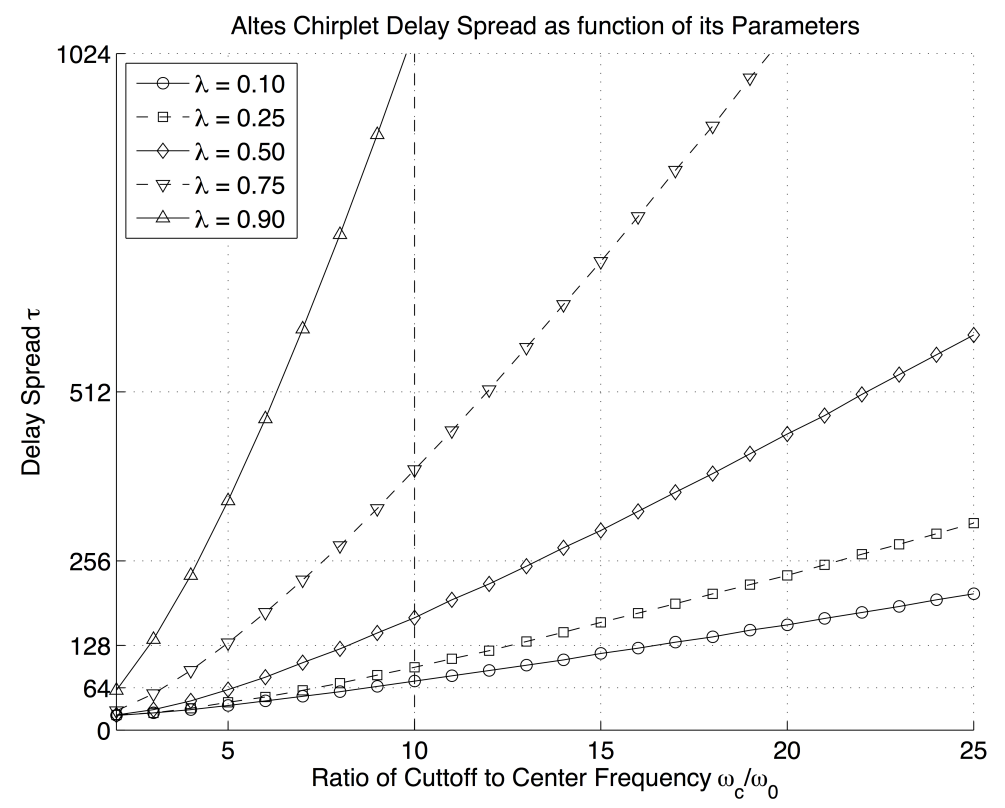

Figure 7. Altes chirplet delay spread $\tau$ as a function of parameters $\left\{\omega_{0}, \lambda\right\}$. The cutoff frequency is fixed at $\omega_{c}=\pi$. The vertical dashed line shows that a 512-point Fourier Transform is appropriate for implementing all chirplets with $\lambda<0.75, \omega_{0}>\frac{\pi}{10}$. See Section 4.10 for details.

\subsection{Choosing the Chirp-Rate Using Oscillation Count \& Discrete Scale Invariance}

We can sensibly bound the chirp rate $\lambda$ by examining the number of oscillations of the chirplet. Consider the chirplets in Figures 2 and 3 . The first example appears to have about two full oscillations of the waveform, while the second has about six. We can provide more precision by counting the number of oscillations of the Altes chirplet over a relevant parameter sweep. Figure 8 shows the measured number of full oscillations of the chirplet for such a sweep. The constraints on center- and cutoff-frequency $\left\{\omega_{0}, \omega_{c}\right\}$ are those in Section 4.8, i.e., $\left\{0<\omega_{0}<\frac{\pi}{4}, \omega_{c}=\pi\right\}$.

We see that for $0<\lambda<1$, a larger $\lambda$ results in a greater number of oscillations, which scales approximately as $-1 / \log (\lambda)$. In fact, it can be seen that the number of oscillations becomes very large for values of chirp-rate close to unity. This is undesirable for two main reasons. Firstly, the well known wavelets such as Meyer, Shannon, Gauss, Morelet, Mexican Hat, etc. all have a small number of oscillations within their delay spread. It has been found that a low number of oscillations, from two or three up to a dozen or so, gives useful results in applications such as data compression and signal analysis. This is because different frequencies and scales can be analysed using a small number of oscillations, by appropriate dilations. This also holds for Altes chirplets. 


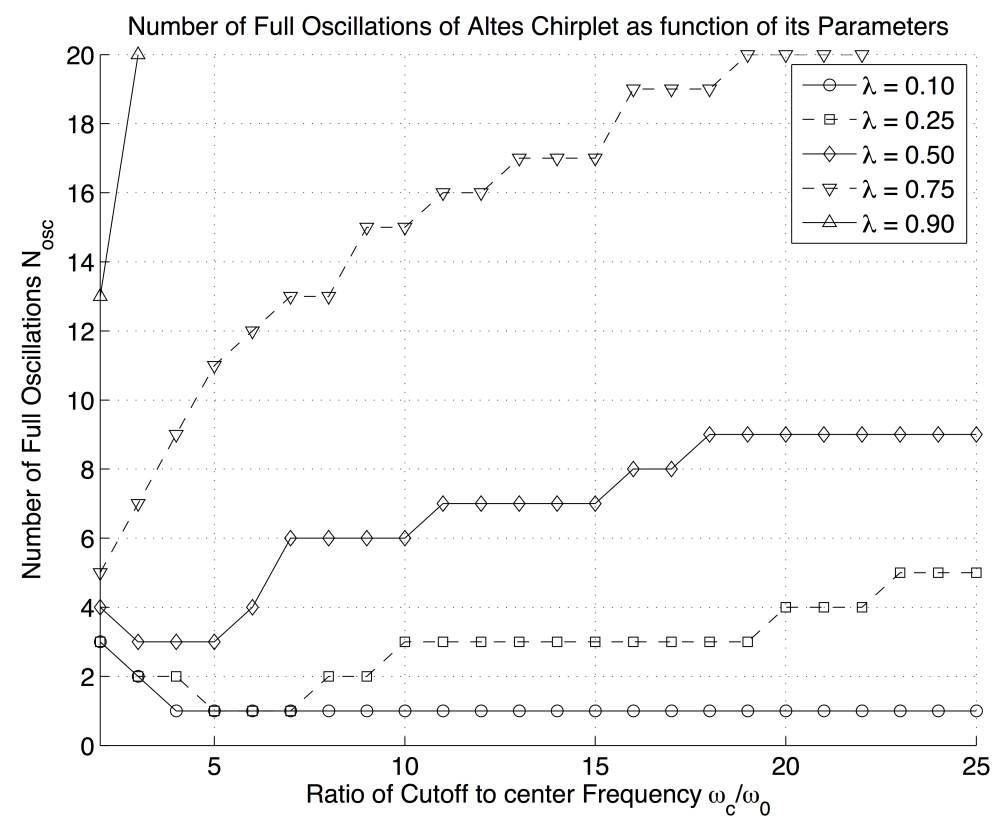

Figure 8. The measured number of full time-domain oscillations of the Altes chirplet within the limits of its delay spread, as a function of its parameters $\left\{\omega_{0}, \omega_{\mathcal{c}}, \lambda\right\}$. See Section 4.9 for details.

A more compelling reason stems from the Altes chirplet property of discrete scale invariance introduced in Section 2.1. Note that discrete scale invariance is different to full scale invariance of the HCT discussed in Section 4.4. Such self-similar signals are invariant under a discrete set of dilations. For log-periodic signals, the ratio of intervals between peaks of the oscillation is constant. This has the advantage that only a few oscillations of the chirplet are necessary for LP-detection, since further oscillations only detect extensions of the log-periodicity at higher or lower (discrete) scales. More simply, we do not need a large number of chirplet oscillations to detect a log-periodicity. A few cycles will do, plus appropriate dilations and scaling, as in the hyperbolic chirplet transform. The implications of this become apparent in Figure 8, which suggests that we need a tighter upper bound than $\lambda<1$. To remain below 20 oscillations of the chirp, we retain the previous parameter bounds but tighten the upper chirp-rate bound to $\lambda<0.75$.

We also propose a lower bound on $\lambda$ based on the oscillation count. Log-periodicity can occur spuriously in noisy data and it is desirable to avoid falsely reporting such events as significant. A deep study on the statistics of random-walk data (time-integrated noise) has shown that the most likely number of spurious oscillations which occur is 1.5 , and that 2.5 can occur with a likelihood as high as $10 \%$ over many realisations [67]. We therefore suggest that usefully parameterised Altes chirplets will have at least 2 oscillations, implying a practical lower bound $\lambda>0.25$ from Figure 8 . Taking both results of this section together, we are imposing $\frac{1}{4}<\lambda<\frac{3}{4}$.

\subsection{Choosing Fourier Transform Size for Discrete-Time Implementation}

When implemented in software or hardware, the HCT must be approximated in discrete-time with a finite number of samples using appropriate time-frequency sampling. To generate the time-domain Altes chirplet $u(t)$ via Inverse FFT (IFFT), we should select a transform size $N_{\text {fft }}$ that is large enough to capture its full delay spread $\tau$. Figure 7 shows the measured delay-spreads of Altes chirplets parameterised for inefficient localisation as in Section 4.9. Since the IFFT is implemented cost-effectively when its size is an integer power of two, the $y$-axis is labeled dyadically, directly allowing the choice of transform size for discrete-time implementation as a function of the parameters. For example, selecting $\frac{\omega_{c}}{\omega_{0}}=10$ and the chirp rate $\lambda=\frac{3}{4}$, it can be seen that a 512-point FFT would be needed for alias-free computation of the Altes time-domain chirplet $u(t)$. Note that increasing $\omega_{c} / \omega_{0}$ 
is used as a proxy for increasing bandwidth as per Equation (11). At these settings, the delay spread increases more or less linearly with bandwidth, requiring higher complexity of implementation.

\subsection{Summary of Altes Chirplet Parameter Selection and Implementation of the HCT}

This Section has exposed several desirable properties of the Altes Chirplet, and how to parameterise it for use in a Hyperbolic Chirplet Transform, e.g., for efficient time-frequency localisation, as summarised in Table 1 . However, it has also been noted that localisationefficiency should be sacrificed for chirping flexibility, and instead we seek parameters that allow wide-band chirping for a selection of chirp-rates with minimised sampling rate and Fourier transform size. A numerical examination of these properties over the parameter space leads to the conclusions of Table 2 for parameterising the Altes chirplet. The snippet of MATLAB ${ }^{T M}$ in Listing 1 shows that the chirplet can be easily implemented in software, given the selected parameters.

Listing 1: This MAtLAB ${ }^{T M}$ function altesChirplet.m generates the Altes Chirplet from its input parameters as per Equation (2). The chirplet is constructed in both frequency- and time-domain representations, respectively, $(U(\omega), u(t))$, the latter being computed via the $N_{\text {fft }}$-point inverse FFT of the former. Please note that in Matlab the function log computes the natural logarithm as desired.

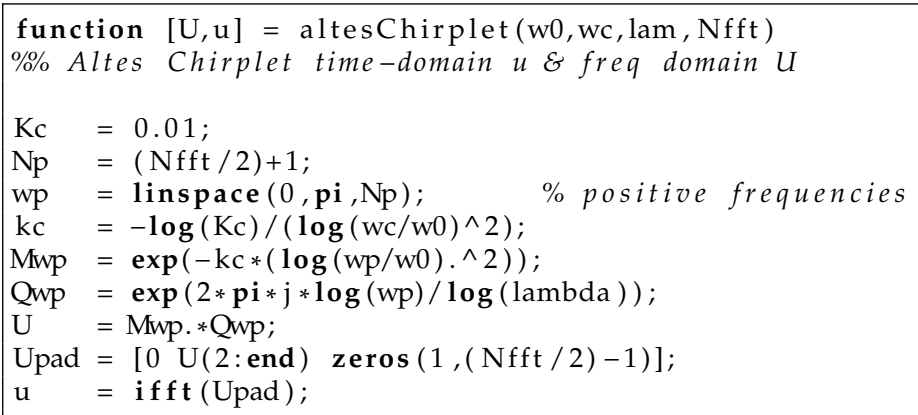

\section{Empirical Results and Discussion}

This section demonstrates the performance of the HCT in detecting log-periodicities buried in noise, in comparison to that of more conventional linear transforms. Section 5.1 describes the experimental setup, Section 5.2 examines the results of the log-periodicity detection and Section 5.3 provides a discussion of these results.

\subsection{Experimental Setup}

In order to demonstrate the applicability of the Altes wavelet to detection of logperiodicities via the Hyperbolic Chirplet Transform, we have constructed a synthetic test signal in which three real-valued hyperbolic chirps are buried in white noise, along with a sine wave. For purposes of transparency, this toy signal $\tilde{x}(t)$ is constructed as

$$
\begin{aligned}
\tilde{x}(t) & =x(t)+s(t)+n(t), \\
\text { where } \quad x(t) & =\frac{7}{10} \frac{u_{1}(t)}{\left\|u_{1}(t)\right\|_{\infty}}+\frac{1}{2} \frac{u_{2}(t)}{\left\|u_{2}(t)\right\|_{\infty}}+\frac{1}{2} \frac{u_{3}(t)}{\left\|u_{3}(t)\right\|_{\infty}} .
\end{aligned}
$$

Sinus $s(t)=\frac{1}{2} \sin \left(\frac{\pi}{3} t\right)$ is tonal interference and $n(t)$ is normally distributed Gaussian white noise added at a level relative to $x(t)+s(t)$ for overall signal to noise ratio (SNR) of $0 \mathrm{~dB}$. This high level of noise is chosen to expose the discriminating power of the various transforms at low SNR. Using the construct of Equation (22) the three chirps $u_{i}(t)$ are derived from scalings and shifts of the fundamental Altes chirplet by

$$
u_{1}(t)=u_{\left\{32, \frac{\mathrm{Nfft}}{2}\right\}}(t), \quad u_{2}(t)=u_{\left\{24, \frac{3 \mathrm{Nfft}}{8}\right\}}(t), \quad u_{3}(t)=u_{\left\{16, \frac{9 \mathrm{Nfft}}{16}\right\}}(t)
$$


with $N_{\mathrm{fft}}=2^{10}$ chosen in the implementation of Listing 1 . The resulting noisy signal $\tilde{x}(t)$ is shown in Figure 9a. For illustration, the clean chirp signal $x(t)$ is also shown without the noise and tonal interference, and the chirp centers of energy ( $\tau_{0}$ from Figure $\left.1 b\right)$ are marked with dashed verticals. We have chosen to analyse $\tilde{x}(t)$ by three methods, a Short Time Fourier Transform (STFT), a Continuous Wavelet Transform (CWT) using the complex Morlet wavelet, and the Hyperbolic Chirplet Transform using an Altes chirplet. The resulting spectrogram/scalograms are shown in Figure $9 b-d$, respectively, in the form of log-magnitude contour charts.
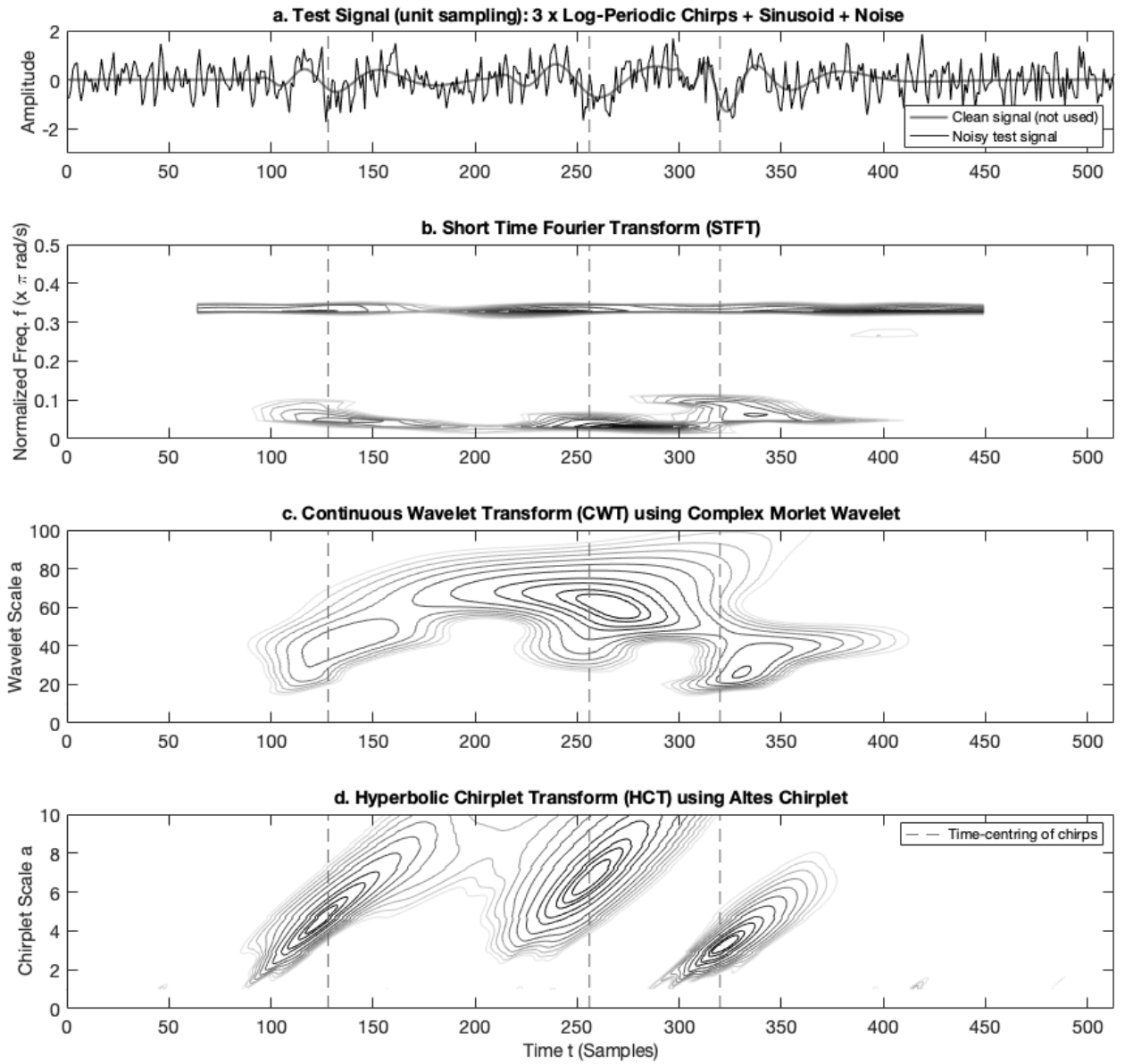

Figure 9. Spectrogram/scalogram detection of log-periodicity in a noisy signal: (a) The noisy signal, (b) Detection by Short-term Fourier Transform (STFT Spectrogram), (c) Detection by Complex Morelet Wavelet Transform (CWT Scalogram) and (d) Detection by Hyperbolic Chirplet Transform (HCT Scalogram). See Sections 5.1 and 5.2 for details.

\subsection{Experimental Results}

Figure $9 \mathrm{~b}$ shows the short-time Fourier transform (STFT) spectrogram of the test signal using a 128-point Fourier Transform and Hamming window. The STFT picks out the sinusoid, which has been injected at $\omega=\frac{\pi}{3}$. This is not detected by the wavelet or chirplet transforms, highlighting the imperative to pick a suitable transform for the problem at hand. Assuming we are only interested in detecting log-periodicities, it is clear that the STFT is relatively poor, as is to be expected from its constant time-resolution at all frequencies. We have implemented a Hamming windowed overlap-add STFT using a 128-point FFT. Note that Overlap-add reduces STFT discontinuities and is available in Matlab as spectrogram.m.

Figure 9c shows some improvement in detecting the LP-chirps, when using a CWT with complex Morlet wavelet. Section 2.4 noted that Morlet wavelets have been reported to exhibit superior performance over other wavelets for chirp detection [46], which is why we 
have chosen a Morlet here as the mother wavelet for the CWT. For fairness of comparison with the (complex) Altes chirplet, we use the complex Morlet, which marginally improves localisation in the scalogram. Furthermore, since the complex Morlet has much narrower time-support than the Altes chirplet at unit scale, we use a higher set of scales in the CWT than the HCT, such that the time resolution of their scalograms are comparable. It is found that we need to choose a set of scales approximately $10 \times$ those of the HCT, as can be seen from the $y$-axes of Figure 9c,d. The CWT scalogram seems to suggest, correctly in fact, that there are three bursts of signal activity over the interval. However, while the result is partially clear around the timing and scaling of signal activity, the localisation of the three LP-chirps is quite poor, and it would be a considerable stretch to conclude that this represents a set of log-periodicities.

Figure 9d shows the Hyperbolic Chirplet Transform scalogram of the test signal using an Altes chirplet. There is a significant increase in sharpness identifying the timing and scaling of bursty signals buried in the noise.

\subsection{Discussion of Results}

Of interest in Figure $9 \mathrm{~d}$ are the three ridges showing that the HCT has captured the benefits of linearising chirps in the time-scale plane, as postulated in $[44,45]$. That is, the formation of linear ridges in the HCT scalogram confirms the presence of log-periodicity. Furthermore, the HCT has correctly isolated the time-centring of the chirps despite high levels of superimposed noise and tonal-interference.

In parameterising the Altes chirplet in this example, we have kept within the bounds of Table 2, selecting $\left\{\omega_{0}, \omega_{c}, \lambda\right\}=\left\{\frac{\pi}{5}, \pi, \frac{1}{2}\right\}$. Focussing on the last parameter, the chirp rate $\lambda$, we note that, in fact, many systems exhibiting discrete scale invariance have a preferred scaling ratio $\lambda=2\left(\Leftrightarrow \lambda=\frac{1}{2}\right)$. For example, in [7], it was observed that the mean field value of $\lambda=2$ is obtained by taking an Ising or Potts model on a hierarchical lattice in the limit of an infinite number of neighbors. Furthermore, we have seen that chirplet parameter $\lambda=\frac{1}{2}$ lies directly within the limits of applicability we found for the HCT $\frac{1}{4}<\lambda<\frac{3}{4}$. We surmise that $\lambda=\frac{1}{2} \Leftrightarrow \lambda=2$ is a natural choice for the chirp-rate. We find that, when making this selection for our mother wavelet, the HCT will nevertheless succeed in detecting chirps generated with different values of $\lambda$. This is because $\lambda$ is a scale ratio for the distance between successive peaks in a log-periodic waveform, while $a$ is the scale ratio within the HCT that serves as a dilation factor, stretching the analysing waveform to find log-periodicities at other chirp rates. This is all to say that fixing $\lambda=\frac{1}{2}$ in the HCT is not seen to limit its use for more general LP-detection.

It is clear from the results of Section 5.2 that the HCT introduced in this paper is a powerful tool for detecting log-periodic signals in noisy measurements. Additionally, it is worth re-emphasising that the underlying properties of the Altes wavelet, together with the parameter tunings introduced here, allow an efficient implementation of the HCT. Building on the mature body of knowledge in wavelet signal processing, we see that the HCT is not just powerful, but also practical.

\section{Summary and Conclusions}

This article has extended the excellent sonar waveform designs of R.A. Altes from the 1970s, making their work on chirping pulses both more accessible, and more widely applicable in the context of wavelet transform analysis. This is motivated by the fact that many signals in nature and in engineering systems display hyperbolic chirping, having logperiodicity, which can be a strong indicator of hierarchical structures and failure dynamics in complex systems. Our approach extends beyond previous attempts at detecting logperiodicity, by introducing the Altes chirp as the mother wavelet in a continuous wavelet transform. We call this new approach the Hyperbolic Chirplet Transform (HCT).

In contrast to previous proposals for log-periodicity detection using Fourier analysis, wavelet transforms, chirplet \& warblet transforms, Constant-Q transforms and the Mellin transform, our approach specifically matches the transform detector to the hyperbolic 
chirps being sought, with an implementation framework which offers tight control over computational complexity.

In order to facilitate this analysis, and as a primary novelty of this work, we propose a re-parameterisation of the original waveform, allowing straightforward specification of a family of chirps in terms of bandwidth, center frequency, and chirp-rate. Our new formulation also simplifies the proof that these chirps constitute a set of admissible wavelets, with the attractive properties of having infinite regularity (i.e., smoothness) and infinite vanishing moments. Furthermore, these chirplets are self-similar at discrete scalings, a remarkable property which delivers scale-invariance when implemented in the hyperbolic chirplet transform.

We have shown in this paper how to choose the parameters of the Altes chirplet for implementation in the HCT. In fact, we propose two choice frameworks for the parameter set, depending on the application at hand. In the first case, we show how to select parameters for optimally efficient time-frequency localisation, which can be useful for signal compression. On the other hand, efficiency can come at the expense of performance in other use cases, giving us reason to seek inefficiently TF-localised chirplets with different properties. We show how to tune the pulse-design settings in these cases also. We put all of this into practice with a synthetic example to illustrate that the HCT facilitates detection of log-periodicity in a noisy signal by linearising its scalogram signature. This feat is not achievable with alternative time-frequency techniques such as the short time Fourier transform or the continuous wavelet transform.

To conclude, it is seen that there is promise in the use of the Altes chirplet and the HCT for improving our ability to detect log-periodic signatures in noisy signals. These theoretical underpinnings for LP-detection can form the basis for applied research in multidisciplinary settings, particularly where there is an imperative to diagnose criticality, and forecast rupture/failure in complex systems. Looking forward, our research is taking us down this more applied route than the theoretical framework of the current study, and there are certainly many real-world applications where the value of the methodology can be quantified more precisely.

Author Contributions: Both authors contributed equally to this work. All authors have read and agreed to the published version of the manuscript.

Funding: This research received no external funding.

Institutional Review Board Statement: Not applicable.

Informed Consent Statement: Not applicable.

Data Availability Statement: Not applicable.

Conflicts of Interest: The authors declare no conflict of interest.

\section{Appendix A. Derivation of the Original Altes Waveform}

Consider waveform $U(\omega)$, which produces a set of echoes when reflected from a target in a sonar detection setting. Depending on the wavelength, its angle of incidence, the velocity and reflectivity of the target and other factors, reflections may be in- or out-ofphase with the incident waveform. By superposition, an echo is thus hypothesised to be the weighted sum of time-integrated (when in-phase) or -differentiated (when out-of-phase) versions of the original signal at different lags.

The goal is to design this transmit signal $U(\omega)$ such that it can be reliably recovered from these $n$ different echo components $V_{n}(\omega)$ under constraints on receiver complexity. Since time-integrated and -differentiated versions of $U(\omega)$ are given in the frequencydomain by $(j \omega)^{n} U(\omega), n \in \mathbb{Z}$, detection can be achieved using a bank of filters $V_{n}^{*}(\omega)$, each matched to one of these energy normalised echo components, written as 


$$
V_{n}(\omega)=\frac{(j \omega)^{n} U(\omega)}{\frac{1}{2 \pi} \int_{-\infty}^{\infty}\left|(j \omega)^{n} U(\omega)\right|^{2}}
$$

Altes' insight was that the complexity of the required filter bank could be constrained if these filters have a constant time-bandwidth product for all values of $n$, i.e., Constant$\mathrm{Q}$ (Section 2.3). This is because the component $V_{n}^{*}(\omega)$ is repeatedly differentiated as $n$ increases, which, from Equation (A1) and the Cauchy-Schwarz inequality, results in bandwidth expansion. If this is compensated by a corresponding compression of the timedomain impulse response, then the required matched-filter complexity remains uniform in $n$. This is true for a bank of Constant-Q filters. Consider matched filters $V_{n}^{*}(\omega)$ which satisfy a scaling constraint defined by $k>1$ :

$$
V_{n}^{*}(\omega) \propto V_{n-1}^{*}\left(\frac{\omega}{k}\right) .
$$

Undesirably, this $k$ introduced by Altes tunes both the bandwidth and chirp-rate of the final waveform, one of the reasons for re-parameterisation.

If $V_{n}^{*}(\omega)$ has root mean square delay-spread $\tau_{n}$ and bandwidth is $B_{n}$, then Equation (A2) gives $B_{n}=k B_{n-1}$ and its inverse Fourier transform gives $\tau_{n}=\tau_{n-1} / k$. Therefore,

$$
\tau_{n} B_{n}=\tau_{n-1} B_{n-1}=\tau_{m} B_{m} \quad \forall m, n \in \mathbb{Z} .
$$

This is a sufficient constant- $Q$ condition on $V_{n}^{*}(\omega)$ to equalise the time-bandwidth products for all $n$, as well as the ratios of centre-frequency to bandwidth. From Equation (A2)

$$
\begin{array}{rcl}
V_{n}^{*}(\omega) & \propto & V_{0}^{*}\left(\frac{\omega}{k^{n}}\right) \\
\Rightarrow \omega^{n} U(\omega) \stackrel{(A 1)}{\propto} & U\left(\frac{\omega}{k^{n}}\right) \\
\Rightarrow \omega^{n} U(\omega) & = & C(n) U\left(\frac{\omega}{k^{n}}\right),
\end{array}
$$

where $C(n)$ is a proportionality constant dependent on $n$ but independent of $\omega$. From relatively simple arguments, we obtain Equation (A6), an explicitly solvable homogeneous functional in $U(\omega)$. By analytic continuation to real values of $n$, and taking the derivative with respect to $n$ of Equation (A6) at $n=0$, and also noting that $C(0)=1$, we obtain

$$
\frac{\mathrm{d} U(\omega)}{U(\omega)}=\frac{C^{\prime}(0)-\log \omega}{\omega \log k} \mathrm{~d} \omega, \omega \neq 0 .
$$

Integration and some simplification leads to the original Altes pulse $U(\omega)$ in Equation (1), designed specifically to have echo components that are easily detectable. This $U(\omega)$ can be verified to satisfy Equation (A6) by choosing

$$
C(n)=k^{n v+\frac{n^{2}}{2}} \exp (-j 2 \pi n c) .
$$

For a more thorough understanding of these concepts, it is worth revisiting the original derivation by Altes [3].

\section{Appendix B. Derivation of the Properties of the Altes Chirplet}

Appendix B.1. Proof of Admissibility as a Wavelet

Proof of Proposition 1 in Section 3. A log-normal random variable $X$ with $\log (X) \sim$ $\mathcal{N}\left(\mu, \sigma^{2}\right)$ has, by definition, probability density function and expected value given by 


$$
\begin{aligned}
& p_{X}(x)=\frac{1}{x \sigma \sqrt{2 \pi}} \exp \left(-\frac{(\log x-\mu)^{2}}{2 \sigma^{2}}\right), x>0 \\
& E[X] \triangleq \int_{-\infty}^{\infty} x p_{X}(x) \mathrm{d} x=\exp \left(\mu+\frac{\sigma^{2}}{2}\right) .
\end{aligned}
$$

The equality (A10) translates to

$$
\frac{1}{\sigma \sqrt{2 \pi}} \int_{0}^{\infty} \exp \left(-\frac{(\log x-\mu)^{2}}{2 \sigma^{2}}\right) \mathrm{d} x=\exp \left(\mu+\frac{\sigma^{2}}{2}\right) .
$$

Replacement $x \rightarrow \omega, \mu \rightarrow \log \omega_{0}$ and $\sigma^{2} \rightarrow \frac{1}{4 \kappa_{c}}$ gives

$$
\sqrt{\frac{2 \kappa_{c}}{\pi}} \int_{0}^{\infty} e^{-2 \kappa_{c} \log ^{2} \frac{\omega}{\omega_{0}}} \mathrm{~d} \omega=e^{\log \omega_{0}+\frac{1}{8 \kappa_{c}}}
$$

and so from Equation (2)

$$
\frac{1}{2 \pi} \int_{-\infty}^{\infty}|U(\omega)|^{2} \mathrm{~d} \omega=\frac{\omega_{0}}{\sqrt{8 \pi \kappa_{c}}} \exp \left(\frac{1}{8 \kappa_{c}}\right)
$$

From Parseval's Theorem

$$
\int_{-\infty}^{\infty}|u(t)|^{2} \mathrm{~d} t=\frac{1}{2 \pi} \int_{-\infty}^{\infty}|U(\omega)|^{2} \mathrm{~d} \omega \stackrel{(A 13)}{<} \infty
$$

proving square integrability by Equation (21). By substituting $x \leftarrow \log \frac{\omega}{\omega_{0}}$ in Equation (21) we obtain

$$
\begin{aligned}
\mathcal{C}_{U} \stackrel{(2)(16)}{=} \int_{0}^{\infty} \exp ^{2}\left(-\kappa_{c} \log ^{2} \frac{\omega}{\omega_{0}}\right) \frac{\mathrm{d} \omega}{\omega} \\
\quad=\int_{-\infty}^{\infty} \exp \left(-2 \kappa_{c} x^{2}\right) \mathrm{d} x=\sqrt{\frac{\pi}{2 \kappa_{c}}}<\infty
\end{aligned}
$$

proving invertability and, therefore, admissibility by Equation (21). As an aside, Equations (A13) and (A14) can be used for normalisation in Equation (23).

Appendix B.2. Proof of Infinite Regularity

Proof of Proposition 2 in Section 3. Using $U(\omega)$ from Equation (2), we define

$$
\begin{aligned}
I_{0} & \stackrel{(24)}{=} \int_{0}^{\infty} \exp \left(-\kappa_{c} \log ^{2} \frac{\omega}{\omega_{0}}\right) \mathrm{d} \omega+\int_{0}^{\infty} \omega^{\alpha} \exp \left(-\kappa_{c} \log ^{2} \frac{\omega}{\omega_{0}}\right) \mathrm{d} \omega \\
& \triangleq I_{1}+I_{2} .
\end{aligned}
$$

Replacing $x \leftarrow \log \frac{\omega}{\omega_{0}}$ gives

$$
\begin{aligned}
I_{2} & =\omega_{0}^{\alpha+1} \int_{-\infty}^{\infty} \exp \left(-\left(\kappa_{c} x^{2}-(1+\alpha) x\right)\right) \mathrm{d} x \\
& =\omega_{0}^{\alpha+1} \sqrt{\frac{\pi}{\kappa_{c}}} \exp \left(\frac{(1+\alpha)^{2}}{4 \kappa_{c}}\right)
\end{aligned}
$$

The limit $\alpha \rightarrow 0$ gives $I_{1}$ allowing us to compute the definite integral

$$
I_{0}=\omega_{0} \sqrt{\frac{\pi}{\kappa_{c}}} e^{\frac{1}{4 \kappa_{c}}}\left[1+\omega_{0}^{\alpha} e^{\frac{\alpha^{2}+2 \alpha}{4 \kappa_{c}}}\right]<\infty
$$

for all finite $\alpha>0$, proving infinite regularity by Equation (24). 
Appendix B.3. Proof of Infinite Vanishing Moments

Proof of Proposition 3 in Section 3. Since $U(\omega)=0, \omega \leq 0$ it suffices by Equation (25) to show that

$$
\lim _{\omega \rightarrow 0^{+}} \frac{\mathrm{d}^{n} U(\omega)}{\mathrm{d} \omega^{n}}=0, \forall n \in \mathbb{Z}^{+} .
$$

It is obvious that the exponential form of the Altes wavelet $U(\omega)$ from Equation (2) renders it infinitely continuously differentiable over the positive frequencies $\omega>0$. While computing these derivatives is cumbersome, by rewriting

$$
U(\omega)=\exp (Q(\omega)), \omega>0
$$

it can be readily found from chain and product rules that they take the form

$$
\frac{\mathrm{d}^{n} U(\omega)}{\mathrm{d} \omega^{n}}=U(\omega) \sum_{p=1}^{n} \sum_{q=1}^{p} a_{p} Q_{p}^{b_{p}}(\omega) a_{q} Q_{q}^{b_{q}}(\omega)
$$

where

$$
Q_{i}(\omega)=\frac{\mathrm{d}^{i} Q(\omega)}{\mathrm{d} \omega^{i}} \stackrel{(2)}{\stackrel{(A 22)}{=}} \frac{c_{i, 0} \log \omega+c_{i, 1}+j c_{i, 2}}{\omega^{i}} .
$$

There are a finite number of terms in the double summation of Equation (A23). This number is independent of $\omega$, as are the integer constants $a_{i}, b_{i}$ and the real constants $c_{i, k}$. These coefficients must be found by computation, but we do not need them to observe that, as $\omega$ shrinks to zero in Equation (A23), the magnitude of $U(\omega)$ shrinks far more quickly than the products of $Q_{i}^{b_{i}}(\omega)$ explode. The former has order of growth $\exp \left(-\log ^{2} \omega\right)$ which dominates the latter, whose order of growth is only $(\log \omega) / \omega^{n}$ at the origin. This dominance holds true for any $n \in \mathbb{Z}^{+}$.

Appendix B.4. Proof of Scale Invariance

Proof of Proposition 4 in Section 3. The continuous wavelet transform of Equation (19) can be written in the frequency-domain, via the Plancharel theorem, as

$$
C_{\psi}(a, b)=\frac{\sqrt{a}}{2 \pi} \int_{\mathbb{R}} S(\omega) \Psi^{*}(a \omega) e^{j \omega b} \mathrm{~d} \omega .
$$

Taking $n=1$ in Equations (A6) and (A8) and using Equation (14) gives

$$
\omega U(\omega)=k^{v+\frac{1}{2}} \exp \left(-j 2 \pi \frac{\log k}{\log \lambda}\right) U\left(\frac{\omega}{k}\right) .
$$

For $k \rightarrow \frac{1}{a}$ in Equation (A26) with $U(\omega) \rightarrow \Psi(\omega)$ in Equation (A25) and $U(\omega)$ given by (2), we obtain

$$
C_{u}(a, b)=\frac{g(a)}{2 \pi} \int_{\mathbb{R}} S(\omega) \omega U^{*}(\omega) e^{j \omega b} \mathrm{~d} \omega
$$

with

$$
g(a) \triangleq a^{1+2 \kappa_{c} \log \omega_{0}} \exp \left(j 2 \pi \frac{\log a}{\log \lambda}\right)
$$

i.e., independent of $\omega$. We have used Equation (8) to replace $v$ in line with the new parameterisation. From the above, it can readily be shown that for any real multiplier $m>0$, we get

$$
C_{u}(m a, b) \stackrel{(A 27)(A 28)}{=} g(m) C_{u}(a, b)
$$


with $g(m)$ independent of $a$ and $b$, proving scale invariance. Please note that at unit scale, $a=1$ and $g(1)=1$. In this case Equation (A27) has the time-domain equivalent

$$
C_{u}(1, b) \stackrel{(19)(22)}{=} s(t) \star u(t-b),
$$

where $\star$ is cross-correlation. In words, we find the HCT coefficients at unit scale by measuring the correlation of signal $s(t)$ with the delayed Altes chirplet, and use Equation (A29) to find the coefficients at other scales.

\section{References}

1. Altes, R.A.; Titlebaum, E.L. Bat Signals as Optimally Doppler Tolerant Waveforms. J. Acoust. Soc. Am. 1970, 48, 1014-1020. [CrossRef]

2. Altes, R.A. Some invariance properties of the wide-band ambiguity function. Acoust. Soc. Am. 1973, 53, 1154. [CrossRef]

3. Altes, R.A. Sonar for generalized target description and its similarity to animal echolocation systems. Acoust. Soc. Am. 1975, 59, 97-105. [CrossRef] [PubMed]

4. Altes, R.A.; Reese, W.D. Doppler-Tolerant Classification of Distributed Targets-A Bionic Sonar. IEEE Trans. Aerosp. Electron. Syst. 1975, 11, 708-724.

5. Skinner, D.P.; Altes, R.A.; Jones, J.D. Broadband target classification using a bionic sonar. Acoust. Soc. Am. 1977, 62, 1239-1246. [CrossRef]

6. Flandrin, P.; Magand, F.; Zakharia, M. Generalized target description and wavelet decomposition [sonar]. Acoust. Speech Signal Process. IEEE Trans. 1990, 38, 350-352. [CrossRef]

7. Sornette, D. Discrete-scale invariance and complex dimensions. Phys. Rep. 1998, 297, 239-270. Extended Version. Available online: http:/ / xxx.lanl.gov/abs/cond-mat/9707012 (accessed on 30 September 2021). [CrossRef]

8. Johansen, A.; Sornette, D. Critical ruptures. Eur. Phys. J. B-Condens. Matter Complex Syst. 2000, 18, 163-181. [CrossRef]

9. Sornette, D. Why Stock Markets Crash: Critical Events in Complex Financial Systems; Princeton University Press: Princeton, NJ, USA, 2017.

10. Zababakhin, E.I. Shock Waves in Layered Systems. Zhurnal Exp. Teor. Fiz. 1998, 49, 642-646. (In Russian); English translation: Soviet Physics JETP 1966, 22, 446-448.

11. Novikov, E. The effects of intermittency on statistical characteristics of turbulence and scale similarity of breakdown coefficients. Dokl. Akad. Nauk SSSR 1966, 168, 1279. [CrossRef]

12. Barenblatt, G.I.; Zel'dovich, Y.B. Intermediate asymptotics in Math. physics. Russ. Math. Surv. 1971, 26, 45-61. [CrossRef]

13. Jona-Lasinio, G. The renormalization group: A probabilistic view. Il Nuovo C. B Ser. 11 1975, 26, 99-119. [CrossRef]

14. Nauenberg, M. Scaling representation for critical phenomena. J. Phys. A Math. Gen. 1975, 8, 925. [CrossRef]

15. Niemeijer, T.; Van Leeuwen, J.M.J. Phase Transitions and Critical Phenomena; London Academic Press: London, UK, 1976.

16. Kapitulnik, A.; Aharony, A.; Deutscher, G.; Stauffer, D. Self similarity and correlations in percolation. J. Phys. A Math. Gen. 1983, 16, L269. [CrossRef]

17. Doucot, B.; Wang, W.; Chaussy, J.; Pannetier, B.; Rammal, R.; Vareille, A.; Henry, D. First observation of the universal periodic corrections to scaling: Magnetoresistance of normal-metal self-similar networks. Phys. Rev. Lett. 1986, 57, 1235. [CrossRef]

18. Fournier, J.D.; Turchetti, G.; Vaienti, S. Singularity spectrum of generalized energy integrals. Phys. Lett. A 1989, 140, $331-335$. [CrossRef]

19. Sornette, D.; Sammis, C.G. Complex critical exponents from renormalization group theory of earthquakes: Implications for earthquake predictions. J. de Phys. I 1995, 5, 607-619. [CrossRef]

20. Saleur, H.; Sammis, C.G.; Sornette, D. Renormalization group theory of earthquakes. Nonlinear Process. Geophys. 1996, 3, $102-109$. [CrossRef]

21. Anifrani, J.C.; Le Floc'h, C.; Sornette, D.; Souillard, B. Universal log-periodic correction to renormalization group scaling for rupture stress prediction from acoustic emissions. J. Phys. I 1995, 5, 631-638. [CrossRef]

22. Sornette, D.; Johansen, A.; Bouchaud, J.P. Stock market crashes, precursors and replicas. J. Phys. I 1996, 6, 167-175. [CrossRef]

23. Sornette, D.; Johansen, A. Significance of log-periodic precursors to financial crashes. Quant. Financ. 2001, 1, 452-471. [CrossRef]

24. Bessis, D.; Geronimo, J.; Moussa, P. Complex spectral dimensionality on fractal structures. J. Phys. Lett. 1983, 44, 977-982. [CrossRef]

25. Derrida, B.; De Seze, L.; Itzykson, C. Fractal structure of zeros in hierarchical models. J. Stat. Phys. 1983, 33, 559-569. [CrossRef]

26. Meurice, Y.; Ordaz, G.; Rodgers, V. Evidence for complex subleading exponents from the high-temperature expansion of Dyson's hierarchical Ising model. Phys. Rev. Lett. 1995, 75, 4555. [CrossRef]

27. Kutnjak-Urbanc, B.; Zapperi, S.; Milošević, S.; Stanley, H.E. Sandpile model on the Sierpinski gasket fractal. Phys. Rev. E 1996, 54, 272. [CrossRef]

28. Gassino, R.; Liu, Y.; Olivero, M.; Vallan, A.; Perrone, G.; Tosi, D. Toward the development of a distributed all-fiber temperature sensor for biomedical applications. In Proceedings of the IEEE International Instrumentation and Measurement Technology Conference Proceedings, Taipei, Taiwan, 23-26 May 2016; pp. 1-5. 
29. Wang, F.; Du, S.; Sun, W.; Huang, Q.; Su, J. A method of velocity estimation using composite hyperbolic frequency-modulated signals in active sonar. J. Acoust. Soc. Am. 2017, 141, 3117-3122. [CrossRef]

30. Thong-un, N.; Hirata, S.; Kurosawa, M.K. Improvement in airborne position measurements based on an ultrasonic linear-periodmodulated wave by 1-bit signal processing. Jpn. J. Appl. Phys. 2015, 54, 07HC06. [CrossRef]

31. Krishnan, S.; Rangayyan, R.M.; Bell, G.D.; Frank, C.B. Adaptive time-frequency analysis of knee joint vibroarthrographic signals for noninvasive screening of articular cartilage pathology. IEEE Trans. Biomed. Eng. 2000, 47, 773-783. [CrossRef]

32. Sheybani, E.; Javidi, G. Advanced image processing for analytics in biomedicine and bioscience. Curr. Trends Biomed. Eng. Biosci. 2017, 6, 1-5. [CrossRef]

33. Hirata, S.; Hachiya, H. Measurement of human body surface displacement by breathing using airborne ultrasound. In Proceedings of the 2015 IEEE International Ultrasonics Symposium (IUS), Taipei, Taiwan, 21-24 October 2015; pp. 1-3.

34. Bahoura, M.; Simard, Y. Blue whale calls classification using short-time Fourier and wavelet packet transforms and artificial neural network. Digit. Signal Process. 2010, 20, 1256-1263. [CrossRef]

35. Xie, H.; Lin, J.; Lei, Y.; Liao, Y. Fast-varying AM-FM components extraction based on adaptive STFT. Digit. Signal Process. 2012, 22, 664-670. [CrossRef]

36. Stanković, L.; Djurović, I.; Stanković, S.; Simeunović, M.; Djukanović, S.; Daković, M. Instantaneous frequency in time-frequency analysis: Enhanced concepts and performance of estimation algorithms. Digit. Signal Process. 2014, 35, 1-13. [CrossRef]

37. Boashash, B.; Ouelha, S. Designing high-resolution time-frequency and time-scale distributions for the analysis and classification of non-stationary signals: A tutorial review with a comparison of features performance. Digit. Signal Process. 2018, 77, 120-152. [CrossRef]

38. Vetterli, M.; Herley, C. Wavelets and filter banks: Theory and design. Signal Process. IEEE Trans. 1992, 40, 2207-2232. [CrossRef]

39. Brown, J.C. Calculation of a constant Q spectral transform. J. Acoust. Soc. Am. 1991, 89, 425-434. [CrossRef]

40. Holighaus, N.; Dörfler, M.; Velasco, G.A.; Grill, T. A framework for invertible, real-time constant-Q transforms. IEEE Trans. Audio Speech Lang. Process. 2012, 21, 775-785. [CrossRef]

41. Mann, S.; Haykin, S. The chirplet transform: A generalization of Gabor's logon transform. In Vision Interface; 1991; Volume 91, pp. 205-212. Available online: https:/ /www.semanticscholar.org/paper/The-Chirplet-Transform-\%3A-A-Generalization-ofGabor-Mann-Haykin/a47d6f83be87c3874b188b3e6a2fd94ab8617189 (accessed on 8 May 2012).

42. Mann, S.; Haykin, S. The chirplet transform: Physical considerations. Signal Process. IEEE Trans. 1995, 43, 2745-2761. [CrossRef]

43. Lu, Y.; Oruklu, E.; Saniie, J. Fast chirplet transform with FPGA-based implementation. Signal Process. Lett. IEEE 2008, 15, 577-580.

44. Mann, S.; Haykin, S. The Adaptive Chirplet: An Adaptive Wavelet Like Transform. In Proceedings of the SPIE's 1991 International Symposium on Optical Applied Science and Engineering, San Diego, CA, USA, 21-26 July 1991.

45. Yin, Q.; Qian, S.; Feng, A. A fast refinement for adaptive Gaussian chirplet decomposition. Signal Process. IEEE Trans. 2002, 50, 1298-1306.

46. Sejdic, E.; Djurovic, I.; Stankovic, L. Quantitative performance analysis of scalogram as instantaneous frequency estimator. Signal Process. IEEE Trans. 2008, 56, 3837-3845. [CrossRef]

47. Peng, Z.; Meng, G.; Chu, F.; Lang, Z.; Zhang, W.; Yang, Y. Polynomial chirplet transform with application to instantaneous frequency estimation. IEEE Trans. Instrum. Meas. 2011, 60, 3222-3229. [CrossRef]

48. Yang, Y.; Zhang, W.; Peng, Z.; Meng, G. Multicomponent Signal Analysis Based on Polynomial Chirplet Transform. IEEE Trans. Ind. Electron. 2013, 60, 3948-3956. [CrossRef]

49. Le, K.N.; Dabke, K.P.; Egan, G.K. Hyperbolic wavelet family. Rev. Sci. Instrum. 2004, 75, 4678-4693. [CrossRef]

50. Abry, P.; Clausel, M.; Jaffard, S.; Roux, S.; Vedel, B. Hyperbolic wavelet transform: An efficient tool for multifractal analysis of anisotropic textures. arXiv 2012, arXiv:1210.1944.

51. Flandrin, P. Time frequency and chirps. In Aerospace/Defense Sensing, Simulation, and Controls; International Society for Optics and Photonics: Washington, DC, USA, 2001; pp. 161-175.

52. Mottin, E.C.; Flandrin, P. On the Time-Frequency Detection of Chirps. Appl. Comput. Harmon. Anal. 1999, 6, 252-281. [CrossRef]

53. Bertrand, J.; Bertrand, P.; Ovarlez, J.P. Transforms and Applications Handbook: Chapter 12—The Mellin Transform; The Electrical Engineering Handbook Series; CRC Press: Boca Raton, FL, USA, 1995.

54. Yang, J.; Sarkar, T.K.; Antonik, P. Applying the Fourier-modified Mellin transform (FMMT) to Doppler-distorted waveforms. Digit. Signal Process. 2007, 17, 1030-1039. [CrossRef]

55. Gluzman, S.; Sornette, D. Log-periodic route to fractal functions. Phys. Rev. E 2002, 65, 036142. [CrossRef]

56. Yiou, P.; Sornette, D.; Ghil, M. Data-adaptive wavelets and multi-scale singular-spectrum analysis. Phys. D Nonlinear Phenom. 2000, 142, 254-290. [CrossRef]

57. Saleur, H.; Sammis, C.; Sornette, D. Discrete scale invariance, complex fractal dimensions, and log-periodic fluctuations in seismicity. J. Geophys. Res. Solid Earth (1978-2012) 1996, 101, 17661-17677. [CrossRef]

58. Wornell, G.W.; Oppenheim, A.V. Wavelet-based representations for a class of self-similar signals with application to fractal modulation. Inf. Theory IEEE Trans. 1992, 38, 785-800. [CrossRef]

59. Yang, J.; Sarkar, T.K. Acceleration-invariance of hyperbolic frequency modulated pulse compression. Digit. Signal Proc. 2008, 18, 228-235. [CrossRef]

60. Altes, R.A. Models for echolocation. In Animal Sonar Systems; Springer: Berlin/Heidelberg, Germany, $1980 ;$ pp. 625-671.

61. Kroszczynski, J.J. Pulse compression by means of linear-period modulation. Proc. IEEE 1969, 57, 1260-1266. [CrossRef] 
62. Geraskin, P.; Fantazzini, D. Everything you always wanted to know about log-periodic power laws for bubble modeling but were afraid to ask. Eur. Financ. 2013, 19, 366-391. [CrossRef]

63. Vetterli, M.; Kovačević, J. Wavelets and Subband Coding, 2nd ed.; Prentice Hall: Englewood Cliffs, NJ, USA; Creative Commons: Boston, MA, USA, 2007.

64. Aguiar-Conraria, L.; Soares, M.J. The continuous wavelet transform: Moving beyond uni-and bivariate analysis. J. Econ. Surv. 2014, 28, 344-375. [CrossRef]

65. Misiti, M.; Misiti, Y.; Oppenheim, G.; Jean-Michel, P. Matlab Wavelet Toolbox User's Guide; 4.15 (Release 2015b) Editon; The Mathworks Inc.: Natick, MA, USA, 2015.

66. Balleri, A.; Farina, A. Ambiguity function and accuracy of the hyperbolic chirp: Comparison with the linear chirp. IET Radar Sonar Navig. 2016, 11, 142-153. [CrossRef]

67. Huang, Y.; Johansen, A.; Lee, M.; Saleur, H.; Sornette, D. Artifactual log-periodicity in finite size data- Relevance for earthquake aftershocks. J. Geophys. Res. 2000, 105, 25. [CrossRef] 